\title{
Gradhiva
}

GRADHIV

Revue d'anthropologie et d'histoire des arts

26 | 2017

En croire ses sens

\section{Voir et entendre le Livre. Une édition marocaine du} Coran

Seeing and Hearing the Book. A Moroccan Edition of the Quran

\section{Anouk Cohen}

\section{(2) OpenEdition}

1 Journals

Édition électronique

URL : http://journals.openedition.org/gradhiva/3438

DOI : 10.4000/gradhiva.3438

ISSN : $1760-849 X$

Éditeur

Musée du quai Branly Jacques Chirac

Édition imprimée

Date de publication : 6 décembre 2017

Pagination : 72-99

ISBN : 978-2-35744-096-8

ISSN : 0764-8928

Référence électronique

Anouk Cohen, « Voir et entendre le Livre. Une édition marocaine du Coran », Gradhiva [En ligne], 26 |

2017, mis en ligne le 06 décembre 2019, consulté le 03 janvier 2020. URL : http://

journals.openedition.org/gradhiva/3438 ; DOI : 10.4000/gradhiva.3438

(c) musée du quai Branly 


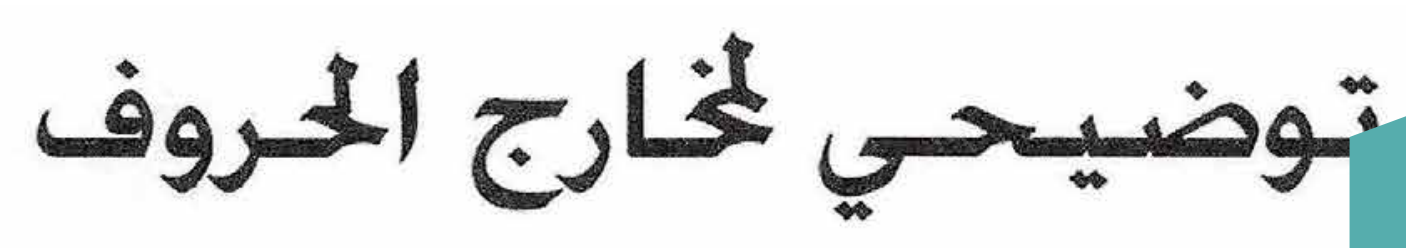

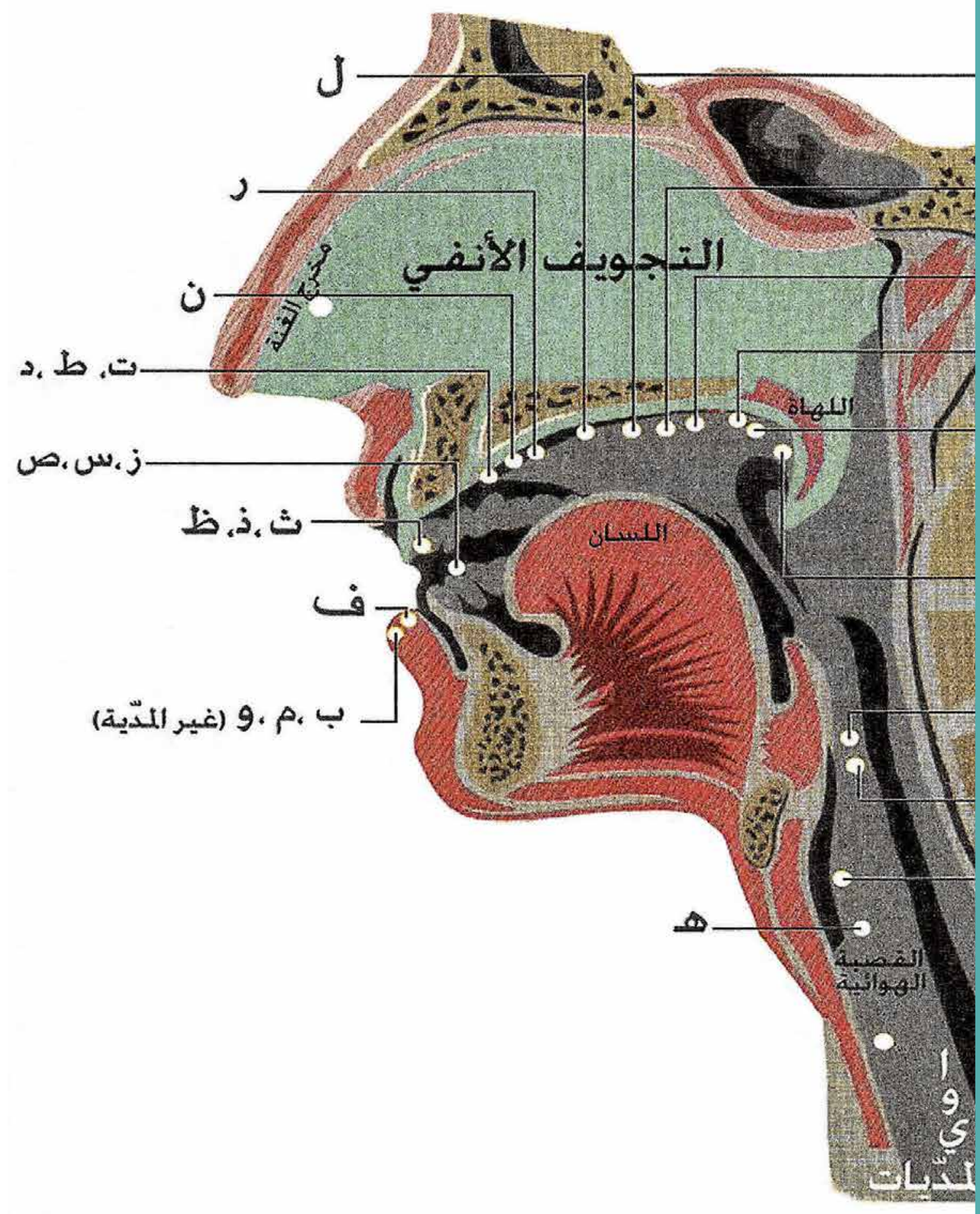

1) 


\section{Voir et entendre le Livre}

Une édition marocaine du Coran

par Anouk Cohen

Cet article porte sur la création en 2010 d'une édition marocaine du Coran qui se distingue par sa méthode de récitation (Warsh), son style calligraphique (maghribî) et les compositions végétales autour du texte. À travers l'étude du processus de fabrication du Livre, telle qu'elle est menée conjointement par les calligraphes, enlumineurs, graphistes et autres, sous le contrôle de clercs institutionnels, il s'agit d'analyser comment les spécificités matérielles du Coran sont mobilisées par la monarchie pour encadrer le rapport sensible des fidèles à la parole divine et assurer sa transmission selon un registre sensoriel proprement marocain et homogène. Fondé sur des pratiques de mémorisation, l'apprentissage coranique met principalement en jeu la vision et l'audition pour faciliter l'incorporation du texte par les pratiquants. Ainsi, plus généralement, cette enquête menée entre 2010 et 2017 questionne la façon dont la relation personnelle et sociale à Dieu est «médiée » par le livre et dont la matérialité de cet objet est pensée pour construire un lien unique au divin : à travers et par le monarque. 
1. Mushaf ("volume») est le terme employé pour ne pas confondre la révélation (qur'ân) avec son support.

\section{Le statut de}

Commandeur des croyants (amîr al-mu'minîn) met l'accent sur l'élection divine du sultan, l'approbation du prophète, l'appui du testament et l'adhésion du peuple. Il est également revendiqué par le sultan d'Oman et, depuis 2014, par le chef de l'État islamique en Irak et en Syrie.
En 2010, le ministre des Habous et des Affaires islamiques annonce la création d'un "Coran marocain ", désormais largement distribué dans les mosquées du royaume. La singularité du mushaf muhammadî $\mathbf{1}$, comme il est encore appelé en référence au roi Mohammed $\mathrm{VI}$, réside dans ses propriétés matérielles. Prenons pour exemples la sourate Al Fatiha (L'Ouverture) et le verset 19 de la sourate $\mathrm{An} \mathrm{Naml} \mathrm{(Les} \mathrm{Fourmis)} \mathrm{tels} \mathrm{qu'ils} \mathrm{sont} \mathrm{reproduits}$ dans le Coran marocain (fig. 1 et 3), d'une part, et dans l'édition saoudienne (fig. 2 et 4), d'autre part. À première vue, le contenu, correspondant à la parole

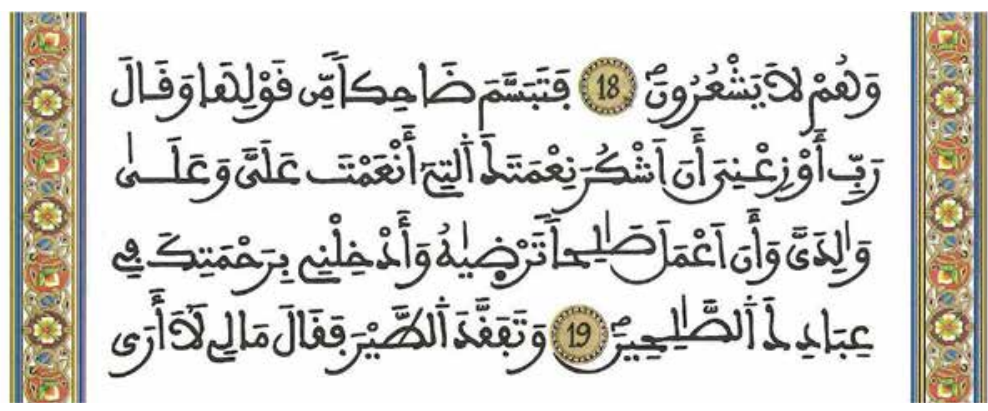

fig: 1

Verset 19 de la sourate An Naml (Les Fourmis) dans le Coran marocain, par Mushaf Mohammadî, Fondation Mohammed VI pour l'édition du Saint Coran, Mohammedia, 2013: 388.
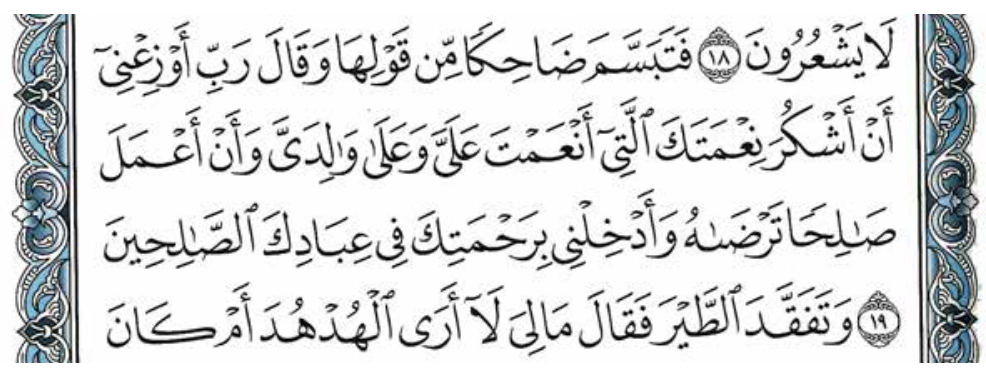

fig. 2

Verset 19 de la sourate An Naml (Les Fourmis) dans l'édition saoudienne du Coran, par Mushaf Al Madinah, Complexe du roi Fahd pour l'impression du Saint Coran, Médine, 2015: 378.

de Dieu, est le même. Cependant, un œil averti notera que la calligraphie (khatt), les enluminures (tadhhîb), les chiffres dénombrant les versets et les signes de vocalisation (tashkîl) varient. «Des détails», disent les éditeurs. «De petits éléments pouvant générer d'immenses polémiques", déclare le ministre, proche conseiller du roi. Autant de distinctions insignifiantes aux yeux des professionnels qui représentent pour lui, en revanche, un enjeu majeur. Quelles intentions y président? Telle est l'interrogation au cœur de la réflexion sur la production d'une spécificité marocaine des façons de voir, d'entendre et de mettre en actes le Coran.

Cette question se pose avec une acuité particulière au moment où le mythe de la commanderie unique des croyants ${ }^{2}$ est mis à mal par l'influence grandissante du Parti de la justice et du développement (islamiste) qui, à la place du roi descendant du Prophète, revendique la sauvegarde de l'islam et de ses valeurs (Zeghal 2005). Après les attentats de Casablanca en 2003, vus comme la mainmise montante d'un islam rigoriste promu par l'Arabie Saoudite wahabbite, Mohammed VI a mis en place une politique religieuse d'envergure visant à définir l'orthodoxie de référence et à renforcer son monopole en tant 
que guide religieux. Ce programme encore tâtonnant défend «l'exclusivité du rite malékite " (discours du trône de 2003), composante essentielle d'un système référentiel réactivé en temps de crise (Tozy 1999; Mouline 2009). Dans ce royaume qui revendique le califat (la position suprême dans l'islam), la relation des fidèles à l'orthodoxie et à l'orthopraxie officielles est en effet au centre du système de légitimation politique (Combs-Schilling 1989; Geertz 1971).

Comme ailleurs par le passé, l'autorité du monarque divin se stabilise et se renforce en prenant appui sur l'imaginaire religieux (Bloch 1983 [1924]) nourri des mythes, des images, des objets et des cérémonies conçus pour figurer le «corps mystique » du roi, surnaturel et immortel (Kantorowicz 1989) ${ }^{3}$. Si le Coran marocain s'inscrit bien dans cet arsenal de symboles, l'étudier invite à dépasser une approche par les seules représentations. L'importance cruciale donnée à la matérialité du livre saint et aux cinq sens qu'elle engage nécessairement conduit à renouer avec l'autre corps, réel ou naturel, de manière à élargir la réflexion à une anthropologie incarnée et sensible de la construction du pouvoir monarchique dans le Maroc contemporain 4 .

Dans la pratique quotidienne des fidèles, le Coran va bien au-delà de son incarnation textuelle. Plus que lu, il est récité, écouté, intégré aux échanges vernaculaires et contemplé sur les murs ornés des mosquées et sur les tableaux décoratifs des maisons. À Casablanca, il est courant de voir de beaux exemplaires du Coran mis en évidence dans le salon des habitations domestiques. Une façon pour les fidèles de décorer leur intérieur et de rappeler aux visiteurs la place que tient la parole de Dieu dans leur maison. De nombreux critères combinés - format, couverture, mise en pages, etc. - créent une infinité d'objets qui correspondent à des usages variés, selon qu'on souhaite garder le livre près de soi, l'apprendre ou le transporter sans l'abîmer. Sans compter les goûts esthétiques des acheteurs, qui s'attachent à leur Coran. Celui qu'ils détiennent n'est pas un simple Coran, c'est le «leur». Une relation tactile, intime et fortement personnalisée (Cohen 2010 et 2012), dans laquelle lire à haute voix, en écouter ou en regarder les versets peut toucher les fidèles et les émouvoir à la manière d'une performance musicale, d'une poésie ou d'un tableau (Zayd 2005). Ils sont ainsi constamment affectés par les formes matérielles du Coran dont ils font une expérience non seulement spirituelle mais aussi pratique, sensorielle et émotionnelle.

De l'enluminure à la calligraphie en passant par la psalmodie, ces ornementations sont censées exalter, rendre présente la parole de Dieu et instaurer une connexion avec le divin (Grabar 1992). Au Maroc, où le roi entend être l'intermédiaire dans cette relation, les gestes associés à ces pratiques, les sens que celles-ci mobilisent et les sensations qu'elles induisent font l'objet d'une réglementation renforcée à l'heure où la libéralisation des biens de salut et le développement des technologies de l'information et de la communication propagent des façons multiples et hétérogènes de s'unir au Verbe. À partir de quelle «gouvernementalité » - au sens de Michel Foucault, soit un mode spécifique d'exercice du pouvoir fondé sur l'induction corporelle d'une discipline intériorisée à travers des procédés matériels (Foucault 1975) ${ }^{\mathbf{5}}$ - le Palais ${ }^{6}$ espère-t-il concrètement contrôler et façonner la subjectivation religieuse?

Répondre à cette interrogation m'a conduite, à la suite d'autres chercheurs (Asad 2001; Hirschkind 2006; Mahmood 2009; Moors 2012), à étudier les formes tangibles de manifestation de l'islam et leurs usages
3. Les Deux Corps du roi (Kantorowicz 1989) repose sur une métaphore énonçant la dualité physique du roi qui posséderait deux corps: I'un naturel, mortel, soumis aux infirmités; I'autre surnaturel, immortel, entièrement dépourvu de faiblesses et incarnant le royaume tout entier.

4. Notons l'absence de travaux sur ces questions, hormis les recherches mentionnées qui ont pris le parti d'une approche historique ou anthropologique des représentations.

5. Se refusant à aborder le pouvoir en termes d'idéologie, Michel Foucault saisit l'État au prisme de ses pratiques à travers le néologisme "gouvernementalité", forgé à partir des termes "gouvernement"

et «rationalité». Le premier se réfère à une conduite destinée à modeler celle des individus, le second suggère que pour contrôler une personne, il faut pouvoir la définir. Par conséquent, l'État va mettre en place des dispositifs matériels visant à discipliner les corps, le temps et les gestes des citoyens dans leurs moindres détails (Foucault 1975).
6. Dans le système politique marocain souvent qualifié de «néo-patrimonial» (un type de domination fortement personnalisé et orienté vers le maintien et la protection des élites en place), le terme "Palais" (encore appelé Makhzen) désigne le cabinet du roi composé de ses conseillers en charge des ministères stratégiques dits "régaliens", incluant celui des Habous et des Affaires islamiques. Le gouvernement et le Parlement détiennent, quant à eux, un pouvoir limité et soumis au contrôle. 


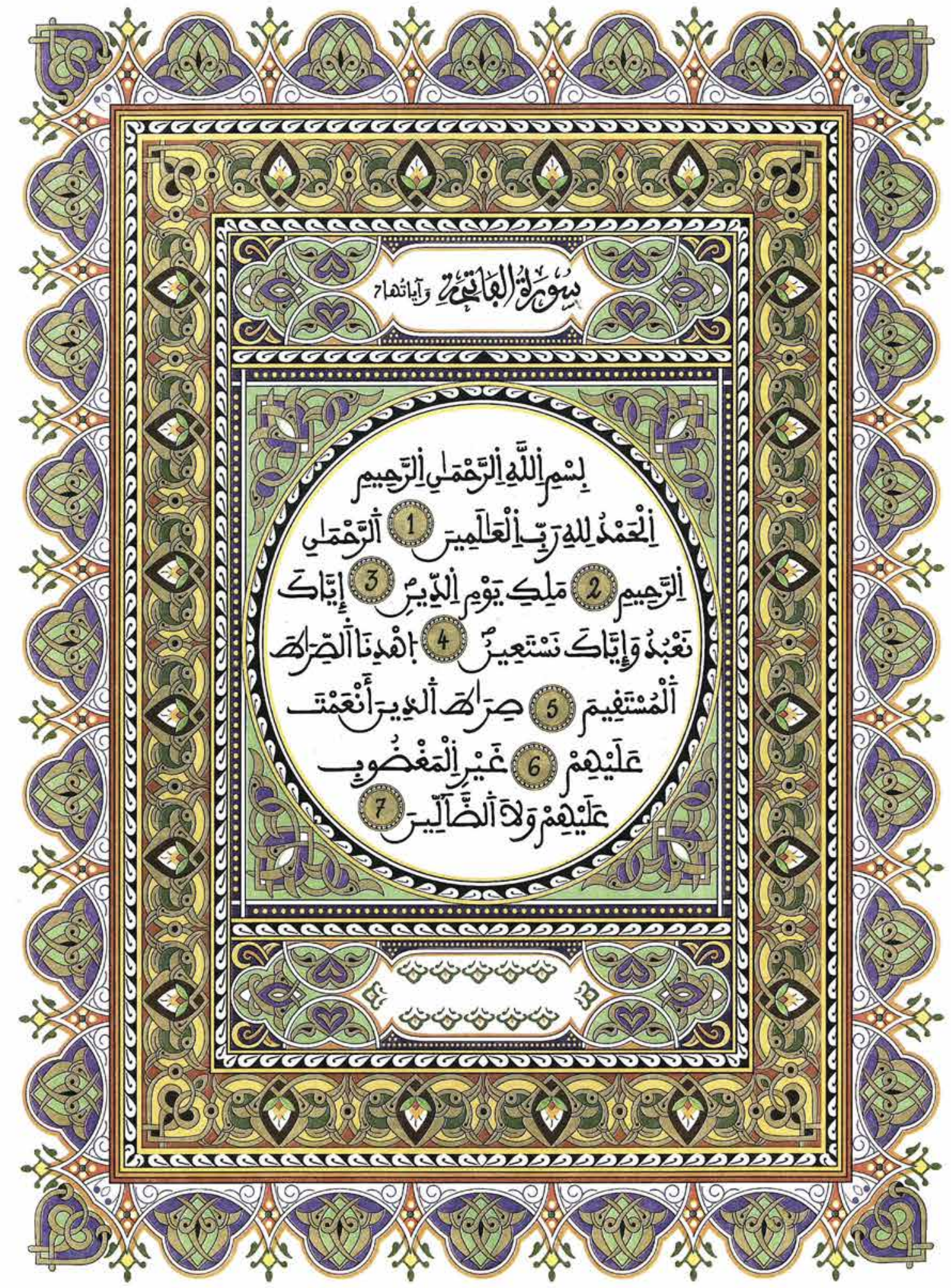


sociaux. Plus précisément, j'ai examiné comment les spécificités matérielles du Livre sont actuellement mobilisées par la monarchie pour tenter d'encadrer le rapport sensible des fidèles au Coran et d'encourager sa transmission selon un registre sensoriel proprement marocain. Ainsi, plus généralement, cette enquête menée entre 2010 et 2017 questionne la façon dont la relation personnelle et sociale à Dieu est «médiée» par le livre et dont la matérialité de cet objet est pensée pour construire un lien unique au divin: à travers et par le monarque.

Le parti pris méthodologique consiste à prendre au sérieux la construction de cette relation en en explorant les dimensions techniques et pratiques (Latour $2006^{7}$ ), à travers une question concrète: comment fabrique-t-on un Coran marocain? Ma démarche, inspirée de la «chaîne opératoire » et inscrite dans une réflexion plus large sur les manières dont les dimensions matérielles des techniques et des objets produisent "des systèmes de sens et de pratiques" (Lemonnier 2004: 3), retrace la genèse d'un livre. II s'agit de saisir comment les acteurs impliqués dans sa fabrication (de sa production à sa distribution dans les mosquées et les écoles) contribuent à l'élaboration et à la définition de l'objet. Ont principalement été étudiés les liens qu'ils entretiennent, les stratégies qu'ils emploient et les matériaux dont ils disposent pour répondre à des objectifs spécifiques et dissonants. Ce sont ces discordances et ratages que j'ai tenté de saisir en centrant l'analyse sur les mécanismes de production d'un «faire croire ${ }^{8}$ » localisé et homogénéisé, servis notamment par les discours autour de l'image de la lettre calligraphiée, des sons émis pendant la récitation et de leurs propriétés.

\section{Fabriquer le Coran marocain}

Erreurs et orthodoxie: le cas exemplaire du mushaf muhammadî

En 2010, la création du Coran marocain est allée de pair avec celle de la Fondation Mohammed VI pour l'édition du Saint Coran, placée sous l'autorité du ministre des Affaires islamiques qui représente la voix du Palais dans ce domaine. Elle est «compétente à titre exclusif pour procéder aux opérations d'enregistrement, d'impression, de publication et de distribution » du Coran (décret de sa création en 2010). L'ouverture de la Fondation fait suite aux polémiques relayées par la presse sur la circulation de livres saints avec des “erreurs» (tahrîf). Selon un éditeur indépendant du quartier des Habous, centre du livre arabe à Casablanca, «il y a beaucoup d'erreurs dans les Corans qu'on vend, des gens qui n'étaient pas du domaine, en voyant que le Coran se vendait bien, se sont mis à scanner des copies et à envoyer les fichiers en Chine. Mais en scannant ils ont fait des erreurs ${ }^{9}$ ". Ils n'ont pas respecté l'ordre des pages communément admis, ont coupé des lignes ou laissé des poussières malencontreuses sur la vitre du scan, susceptibles d'être prises pour des signes diacritiques ${ }^{10}$.

Des fidèles mécontents n'ont pas hésité à se rendre dans la délégation du ministère des Affaires islamiques de leur région, munis d'exemplaires corrigés au crayon à papier. «lls nous demandent de réagir, déclare le directeur de la fondation, alors Sa majesté s'est engagée à distribuer dans les mosquées un Coran saint certifié »: le sien, marqué du sceau royal et garant de l'orthodoxie. L'erreur apparaît comme l'argument politique justifiant la création d'une édition marocaine du Coran ${ }^{\mathbf{1 1}}$.
7. Bruno Latour (2006) invite à penser la construction d'un projet politique comme celle d'un bâtiment en portant l'attention sur ses fondations, les matériaux employés pour les bâtir et les gestes effectués au cours du processus.

\section{8. "J'entends par} "croyance" non l'objet du croire (un dogme, un programme, etc.), mais l'investissement des sujets dans une proposition, l'acte de l'énoncer en la tenant pour vraie [...]. Or, la capacité de croire semble partout en récession dans le champ politique [...]. Par ce lien, la politique explicitait son rapport de différence et de continuité avec la religion. Mais la volonté de "faire croire" dont vit

l'institution fournissait dans les deux cas un répondant à une quête d'amour et/ou d'identité. "

(Certeau 1990: 260)

9. Les entretiens cités dans le présent article ont été menés intégralement en français, intégralement en arabe, ou dans un mélange des deux langues. Dans les deux derniers cas, la traduction a été réalisée par l'auteur.

\section{ci-contre}

fig. 3

La sourate Al Fatiha (L'Ouverture) dans l'édition marocaine du Coran, par Mushaf Mohammadî, Fondation Mohammed VI pour l'édition du Saint Coran, Mohammedia, 2013: 2
10. L'écriture arabe "comporte vingt-huit lettres, mais elle ne dispose en réalité que de quinze caractères [...]. Pour distinguer les consonnes notées par une même lettre, on utilise des points simples, doublés ou triplés, placés sur ou sous la lettre [...]. L'arabe n'indique

que les trois voyelles Iongues au moyen des lettres [...]. Pour indiquer les trois voyelles brèves il a emprunté au syriaque trois signes qui sont suscrits ou souscrits" (Troupeau, site internet: http://classes.bnf.fr/ ecritures/arret/

lesecritures/arabe/02.htm, consulté

le 19 février 2017) afin de doubler les lettres ou de marquer un arrêt sur l'une d'elles. L'expression commune "signes diacritiques" englobe l'ensemble de ces points/signes.

11. En Égypte, c’est le concept de «pollution sonore" qui a servi de prétexte pour standardiser l'appel à la prière (adhân), au cœur de la politique religieuse du milieu des années 2000 (Farag 2009). 
12. La conception de la Révélation dans l'islam impose des contraintes spécifiques dans la reproduction et la diffusion du livre saint. L'adoption tardive de l'imprimerie dans le monde musulman, près de quatre siècles après son avènement en milieu chrétien, en est l'illustration.

Cette résistance a tenu principalement à l'opposition des clercs qui craignaient que le Coran ne soit altéré par sa reproduction technique et que la standardisation de l'imprimé ne bouleverse le système de transmission du savoir au fondement de leur autorité (Robinson 1993). Dans le cadre de l'apprentissage coranique, l'écrit, associé à un ensemble de pratiques orales, est un aidemémoire qui permet son incorporation correcte. Jusqu'à aujourd'hui, la quasi-totalité des livres du Coran produits dans le monde ont été écrits à la main, scannés, retouchés puis imprimés après avoir été vérifiés et certifiés. C'est la manière que les producteurs ont trouvée pour répondre au changement des technologies et préserver l'exactitude du Coran. Ce mode de fabrication - qui n'est pas sans rappeler la lithographie permet de conserver les caractéristiques physiques d'anciens manuscrits comme le style calligraphique et les marques de vocalisation, qui soutiennent jusqu'à aujourd'hui la lecture et la récitation coraniques (Cohen 2016).
13. Warsh est l'appellation donnée à l'une des sept lectures canoniques (qirâ'a, un terme qui correspond à la fois à l'acte de lire et de réciter) Les variantes concernent des points de vocalisation, des emplacements de pause ou de fin de verset ainsi que des détails de prononciation, à l'origine de manières distinctes de rendre le texte à l'oral. Aujourd'hui, à l'exception de larges territoires d'Afrique occidentale et centrale où la récitation selon Warsh est répandue, c'est la récitation selon Hafs, qui est la plus connue.

14. De style coufique connu pour sa grande clarté, utilisé dans les premiers manuscrits coraniques, le maghribi développé au $x^{e}$ siècle pour remplacer l'écriture naskhî

issue des territoires centraux du califat, se caractérise par un style anguleux et géométrique, différent du naskhî, souple et arrondi adopté pour l'imprimerie, la machine à écrire et l'ordinateur. Par conséquent, cette dernière écriture est la mieux connue.

15. Il s'agit d'une édition réalisée en 1929 pa un célèbre faqîh (juriste) et calligraphe réputé pour son écriture et sa connaissance du Coran dont les droits appartenaient jusqu'à une période récente à Dar al-mushaf al-sharîf, installée au Caire

(Fawzi 1990). Nommé Zwiten, ce faqîh était professeur à al-Qarawiyyîn, une importante université d'étude islamique à Fès.

\section{ci-contre}

fig. 4

La sourate Al Fatiha

(L'Ouverture) dans l'édition saoudienne du Coran, par Mushaf Al Madinah, Complexe du roi Fahd pour l'impression du Saint Coran, Médine, 2015: 1
En principe, le processus d'estampille du Coran est strict. Alors que la Bible est soumise aux droits d'auteur (ou plutôt de traducteurs), le livre du Coran ne répond à aucune réglementation officielle. Suivant le protocole défini dans les années 1920 au Caire par Al-Azhar, principale université d'études islamiques, un contrôle doit normalement être réalisé à chaque étape de production. Mais ce protocole est respecté de manière plus ou moins rigoureuse en fonction de la réglementation en vigueur dans chaque pays (Albin 2005). Celle-ci était inexistante au Maroc avant 2011, tandis qu'en Égypte et en Arabie Saoudite les éditions sont contrôlées par des oulémas ('ulama, les clercs de l'islam) depuis plus d'un demi-siècle. Cela explique, selon le directeur de la fondation, «les erreurs dans le texte même: la lettre qui doit être prolongée comme ça ou comme ça. C'est très précis ». C'est pourquoi, outre le conseil d'administration dirigé par le ministre des Affaires islamiques, la fondation s'est dotée d'un «organe scientifique » composé de clercs.

Ainsi, si pour les éditeurs, il existe des livres mal conçus par des libraires incompétents soucieux de produire au plus vite un maximum d'exemplaires, l'appréciation du directeur de la fondation dénonce quant à elle les éditions produites sans le contrôle de clercs institutionnels et dont le texte mal écrit peut être entaché d'hérésie ${ }^{\mathbf{1 2}}$. À ce moment-là, ce qui est qualifié «d'erreur » pose question. Le directeur explicite: «Il faut interroger les membres de l'organe scientifique qui connaissent bien les règles d'écriture. » "L'erreur » se rapporterait donc à un usage hétérodoxe, contraire aux normes établies par les clercs de la fondation. Quelles sont-elles et à quelles discussions ont-elle donné lieu, notamment avec le calligraphe (khattât), l'enlumineur et le graphiste?

\section{À la recherche d'originaux... du Palais}

En 2006, le ministre des Affaires islamiques demanda à un célèbre calligraphe marocain d'écrire le mushaf mohammadî dans le respect des règles de la récitation dominante au Maroc (Warsh ${ }^{\mathbf{1 3}}$ ) et de la calligraphie «maroco-andalouse» (maghribî ${ }^{\mathbf{1 4}}$ ). Celui-ci copia durant des mois les versets sur des planches de papier selon le «Coran de Zwiten ${ }^{15}$ » considéré par les spécialistes de la Bibliothèque royale comme la plus ancienne copie lithographiée au Maroc avec la numérotation des versets. Jusqu'à aujourd'hui, c'est le modèle original majoritairement reproduit par le scan ou la copie manuscrite (Cohen 2016). II s'agit d'un livre canonique différent de l'édition standard, conforme à la copie égyptienne mise au point à al-Azhar en 1924 dans un autre style de calligraphie (naskhî) et un autre mode de récitation (Hafs) souvent qualifiés au Maroc d'«orientaux».

Tout comme le choix de la copie, celui de l'artiste n'est pas anodin: devenu «calligraphe du Palais» sous le règne d'Hassan II (1961-1999), celui-ci a appris le maghribî en reproduisant les écritures inscrites dans les plus anciennes mosquées du royaume. À la suite de son père, il a rédigé les décrets et déchiffré les manuscrits des archives royales et sultanniennes, illisibles pour un œil non entraîné. Ce travail au long cours lui a permis d'approfondir ses connaissances de la calligraphie maroco-andalouse dans le respect des conventions adoptées par la monarchie. C'est donc à lui que Mohammed VI, comme l'avait fait son père Hassan II, s'est adressé pour copier «son Coran ». Contrairement au mushaf hassani voué à être offert aux invités 


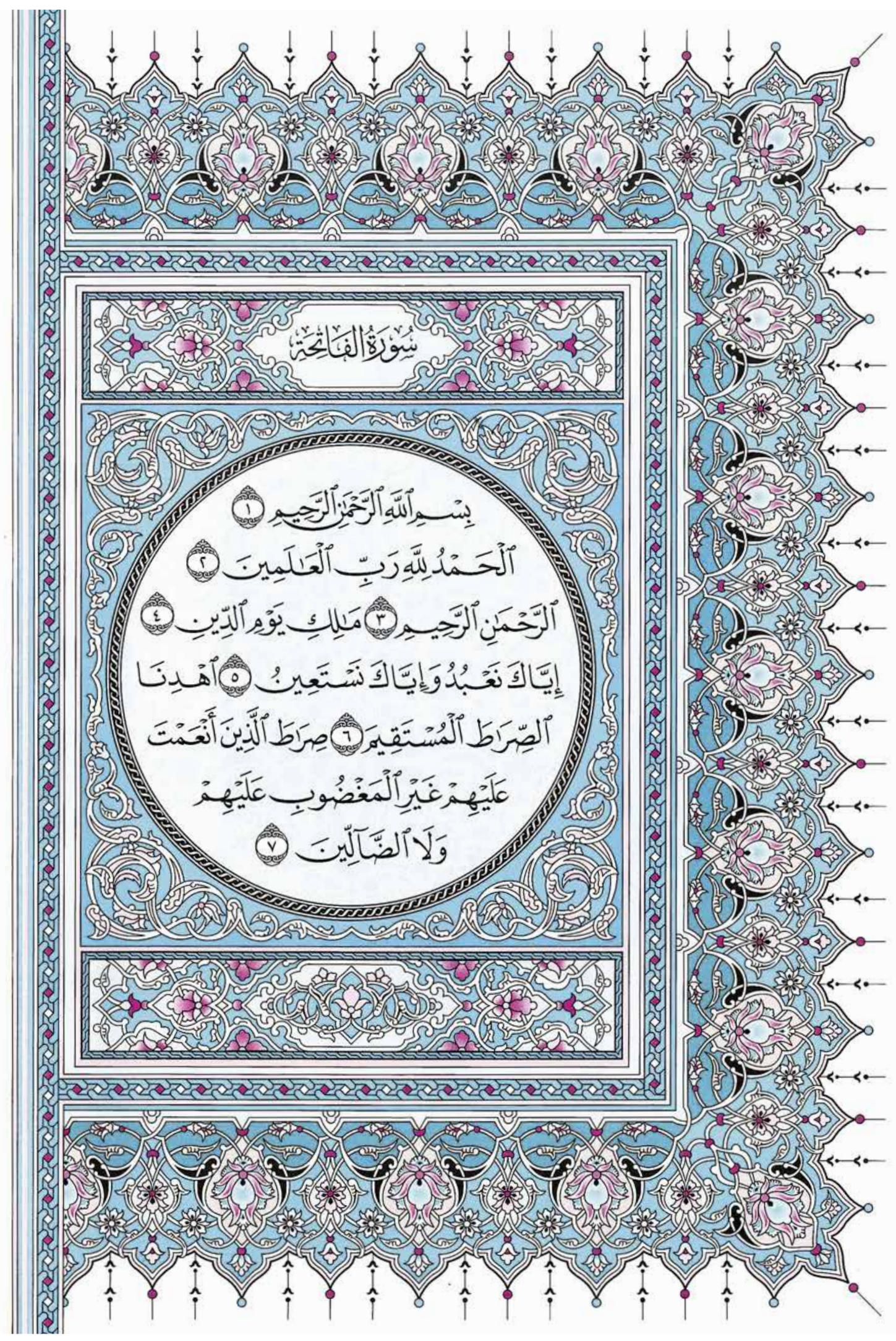




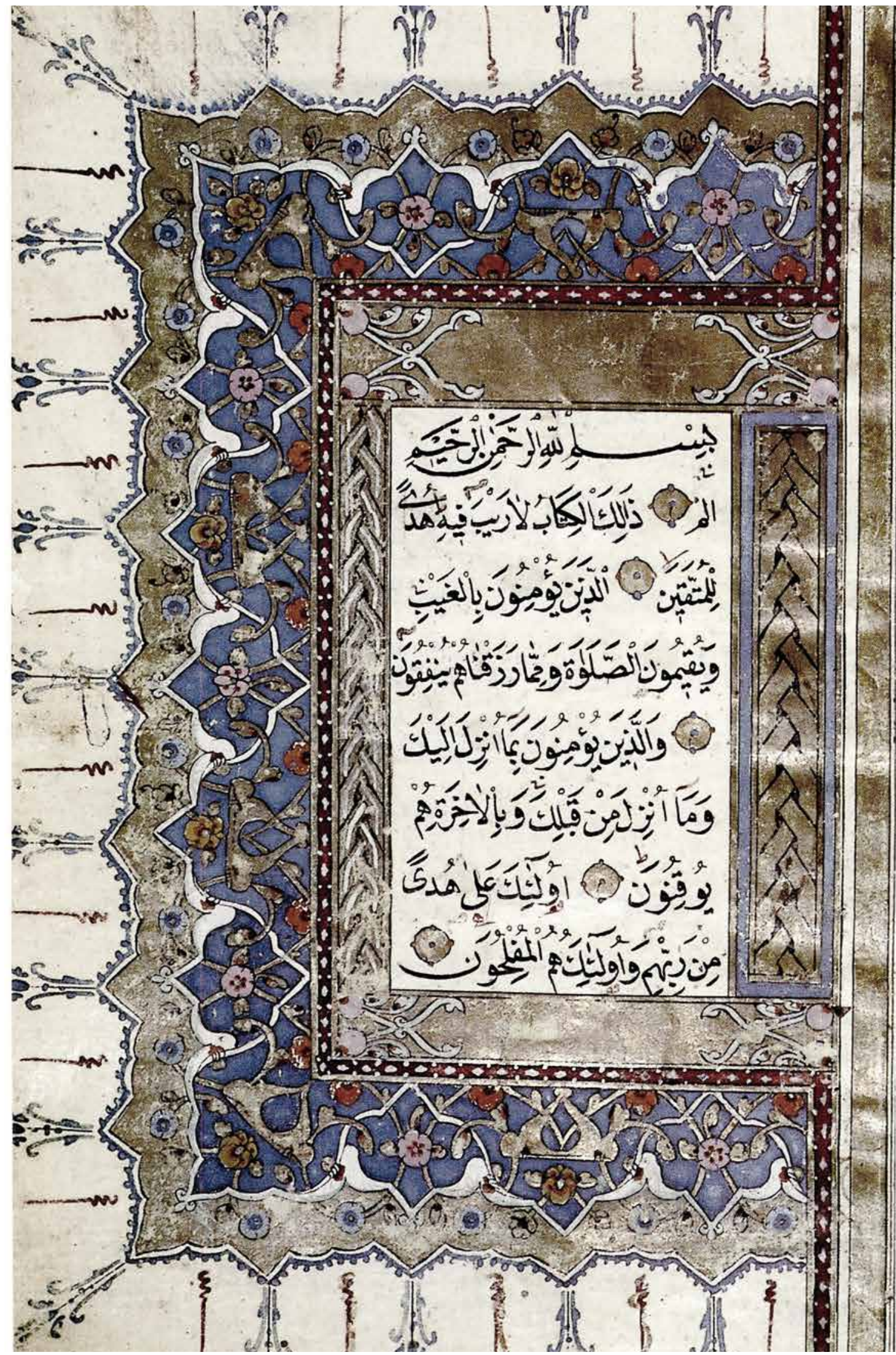


de marque, le mushaf mohammadî a fait l'objet d'une production sérielle et industrielle. Pour le nouveau roi, le calligraphe devait produire un livre inédit: “L'écriture devait être encore plus belle, plus raffinée», m'explique-t-il.

Une fois le travail terminé, les planches calligraphiées ont été remises à deux clercs choisis par le Palais et connus pour leur maîtrise de la calligraphie et de la récitation. L'un, originaire de la région de Fès, âgé d'environ 80 ans, a «appris par cœur le Coran dans toutes ses versions [entendre les sept récitations marocainnes] » suivant la méthode traditionnelle du lûh (planche coranique qui combine l'écriture et la récitation) employée dans l'ensemble du monde islamique, en particulier au Maroc (Eickelman 1986). Son père et son grand-père, décrits comme des shorfa (descendants du Prophète), avaient mémorisé le Coran avant lui dans son intégralité. Ancien enseignant à la faculté des lettres de Rabat et auteur d'ouvrages célèbres sur les récitations du Coran, il a fait partie du renommé comité de vérification d'Al-Azhar. L'autre, un de ses anciens élèves, âgé d'une soixantaine d'années et également originaire de Fès, maîtrise seulement, pour sa part, les récitations les plus répandues. Moins connu que son aîné, il jouit néanmoins d'une notoriété établie grâce à son étude des usages du lûh dans les écoles coraniques (madrasa) du royaume, commandée par le Palais. Tout comme son maître, il est l'héritier et l'un des derniers témoins des façons qdim («ancien», «patrimonial») de transmettre le Coran selon des modalités de récitation et d'écriture propres au pays.

\section{Formes, normes et discordances}

Forts de leur expérience et de leur renommée au Maroc et au sein du Palais, les deux clercs n'ont pas hésité à signaler les erreurs commises par le calligraphe. «Cela manque de précision », ont-ils déclaré avant de proposer d'écrire un nouveau Coran avec l'aide d'un clerc supplémentaire nommé par le ministre suivant les mêmes critères qui avaient présidé au choix des premiers: son ancrage dans un système local de pratiques d'écriture et de récitation. Les séjours fréquents de ce dernier en Arabie Saoudite et les prêches qu'il prononce lui valent cependant d'être qualifié de wahabbite par ses pairs, jugeant qu'il a été choisi dans l'idée de le pousser, lui et ses disciples toujours plus nombreux, à se rapprocher du malékisme. Enfin, l'associer à l'organe scientifique de la fondation en dépit de son attachement à une doctrine différente de celle en vigueur dans le royaume renforcerait l'image d'un régime tolérant et ouvert.

Pendant plusieurs semaines, le calligraphe a rencontré les clercs, muni des planches réécrites suivant leurs corrections, portant principalement sur la forme des lettres. "Par exemple, dit l'aîné des clercs, dhalika ["cela"], on ne peut pas l'écrire comme ça dans le Coran [il trace le mot sur une feuille (fig. 6)], on ne doit pas laisser de vide. Le trait ne doit pas être aussi long,

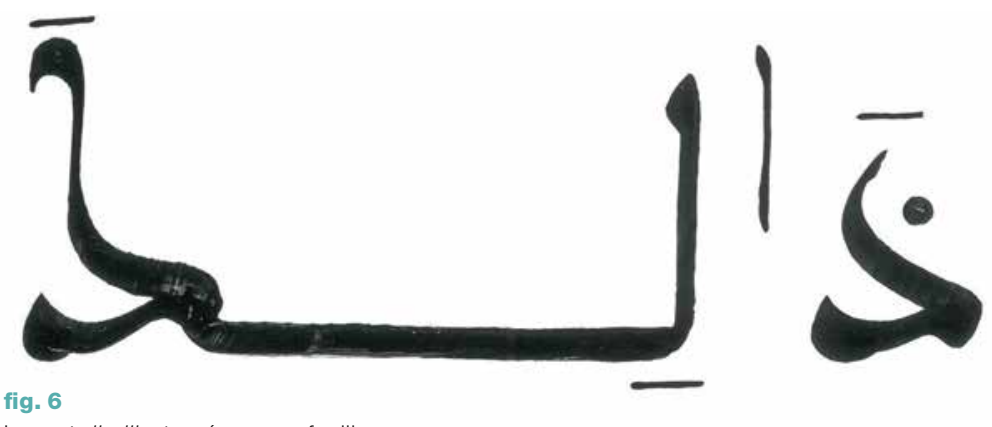

\section{ci-contre}

fig. 5

Coran calligraphié en

écriture naskhî par Ibrahim

Sami en 1785. Plusieurs

cadres entourent le texte

coranique. Les deux

premiers représentent

des tresses, les autres

des entrelacs floraux.

Origine probable:

Moyen-Orient, xvIII ${ }^{\mathrm{e}}$ siècle, in Sijelmassi 1987: 220. 
même si ça ne change rien à la signification. C'est pour ne pas lire de manière excessive.» Car tout se prononce dans la calligraphie maghribî («maroco-andalouse»). La longueur du trait de la lettre équivaut à l'intensité du son à émettre quand on la vocalise et le trait lui-même est considéré comme un «vide» susceptible d'être rempli. C'est pourquoi, «l'espace» entre les lettres a été scrupuleusement vérifié par les clercs qui ont défini une taille propre à chacune d'elles, où s'exprime par ailleurs le talent de l'artiste: «Quand tu prends la lettre, dit le calligraphe, tu la transformes décorativement, tu l'écris à ta façon. Dans la calligraphie maroco-andalouse, il n'y a pas de règles [de mesure] comme dans l'écriture orientale. Chaque calligraphe connaît la forme de la lettre mais quand il veut la réaliser dans le tableau, il la trace selon la forme de ce tableau. " Les clercs ont également interdit les formes jugées confondantes pour le lecteur: "La queue du ra par exemple ne devait pas être si longue et toucher la lettre suivante, explique le calligraphe, [de la même manière] le mot al-hamdu li-I-llâh, je vais l'écrire ici en décoratif (fig. 7); et là (fig. 8), en pensant au Coran. Dans le premier dessin, on voit la forme,

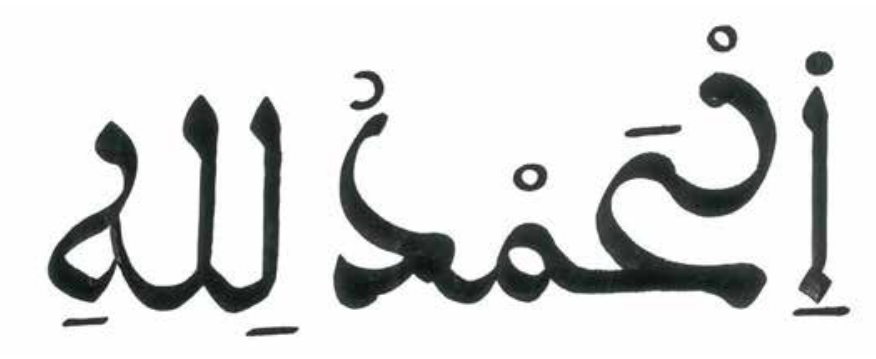

$\mathrm{fig} .7$

Le mot al-hamdu li-l-llâh écrit "en décoratif "

par un calligraphe.

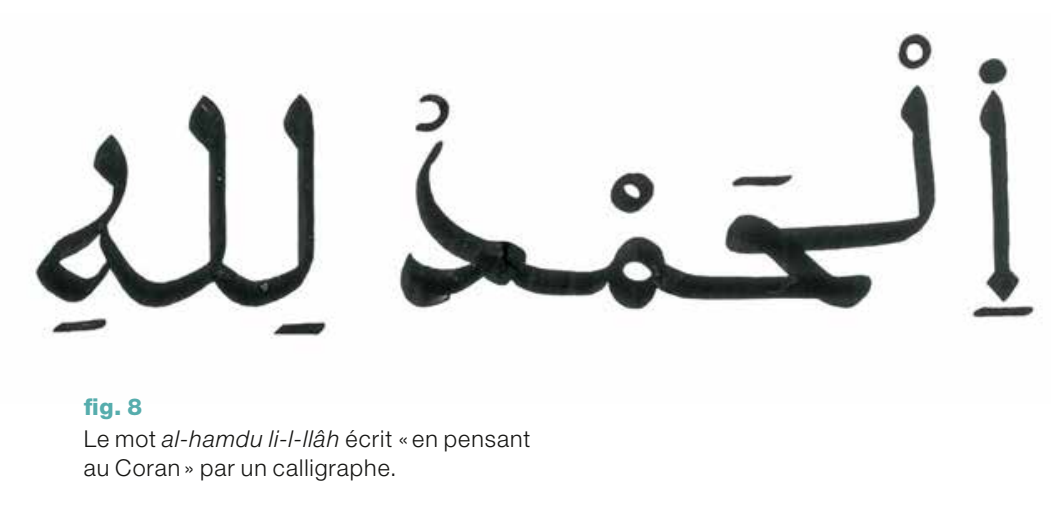

on voit la beauté [...]. Mais, concède-t-il, comme les lettres sont serrées, c'est difficile à lire. C'est pourquoi, poursuit-il, dans le deuxième dessin, on laisse la distance pour le lâm, et on commence à écrire le hâ'. Chaque lettre prend sa place.»

Ces règles d'écriture ont fait l'objet de longues discussions entre les clercs qui ne s'accordaient pas toujours sur la norme à adopter: "L'un disait que l'accent devait être placé juste au-dessus de la lettre et pas à côté,même d'un millimètre, alors que pour moi non", dit un clerc. Pour trancher, ils ont comparé les planches collectées des années plus tôt par l'un d'eux dans les écoles coraniques du pays. Quand ils ne parvenaient pas à s'entendre sur la 
forme d'une lettre, les clercs choisissaient celle qui revenait fréquemment dans les planches, considérées comme le dispositif de transmission le plus fiable car il repose sur la relation personnelle entre le maître et l'élève. Pour établir et fixer les normes d'écriture, ils ont également imposé la taille du kalam («stylo»), outil central et hautement personnalisé dans l'art de la calligraphie: « «l ne devait pas dépasser un millimètre. Ça a été un peu difficile: j'étais tellement habitué à faire les lettres librement. En plus, j'utilisais le kalam en roseau, donc pour lui donner une taille d'un millimètre ça a été dur. J'ai fini par écrire au stylo plume qui, lui, ne se déforme pas », déclare le calligraphe.

L'artiste pour sa part se faisait cependant une toute autre idée de sa tâche, soucieux de reproduire le Coran de sa plus "belle écriture". "Dans son âme, dit-il, le calligraphe trace la lettre dans un but décoratif." Il vise le beau. À plusieurs reprises, il a refusé d'introduire les corrections des clercs: «lls disaient que c'était une erreur, qu'il fallait mettre plus ou moins d'espace entre les lettres. Ils disaient que mon style d'écriture n'était pas clair, qu'il ne respectait pas le Coran, mais c'est eux qui ne le respectaient pas en me demandant de rendre mon écriture moins belle! " Lui et les clercs défendent deux styles d'écriture opposés: l'un caractérisé par sa clarté, l'autre par le raffinement du trait. «Finalement, raconte un clerc, le calligraphe a reconnu que nous avions raison", et il a obéi aux règles imposées.

Une fois les planches terminées, ce fut au tour de l'enlumineur de dessiner les compositions végétales autour du texte. Ce travail n'a pas été supervisé par les clercs qui le jugeaient accessoire, contrairement au ministre pour qui la structure des contours et les couleurs des enluminures devaient "rappeler le goût des Marocains", comme le dit aussi le directeur de la fondation. Singularités esthétiques que l'on retrouve dans les anciennes mosquées du royaume dont l'enlumineur a recopié les décors par imitation des gestes de son père qui avait rejoint le Palais avant lui. Une familiarité entre le lieu de culte et le livre a donc été recherchée. De ces deux espaces de prière se dégage alors un «air connu». Prier dans la mosquée ou «dans» le livre doit provoquer la même sensation: se sentir simultanément proche de Dieu et du monarque. La matérialité des lettres et des enluminures donne au roi une présence physique et en vient à signifier intrinsèquement la sacralité du Coran et la sienne.

Après que l'enlumineur eut fini de réaliser les décorations sur des planches à part, un graphiste les monta avec le texte en veillant à produire un "livre net, sans taches", provoquant parfois des désaccords entre lui et les clercs, le calligraphe et l'enlumineur. Un clerc fit remarquer qu'un signe avait été supprimé: «ll ne s'agissait pas d'une tache mais d'un accent»; le calligraphe se plaignit qu'une lettre ainsi reformatée ne répondait plus aux règles esthétiques de la calligraphie maghribî: "Elle était trop arrondie comme l'écriture orientale»; enfin, l'enlumineur remarqua: «Tu ne peux pas faire passer cette branche sur celle-ci, les ornementations marocaines s'inspirent des règles de la nature. " Face à ce genre de dilemme, le graphiste trancha toujours en faveur des clercs, suivant les instructions strictes du ministre des Affaires islamiques.

Ces observations mènent à trois constats. Les acteurs impliqués dans la fabrication du Livre se représentent de manière différente, voire contradictoire, ce à quoi doit ressembler le Coran marocain, de surcroît celui du roi. Par ailleurs, l'objet, considéré comme le contenant d'une parole révélée, est modulable selon des règles changeantes, ici redéfinies pour figurer une 


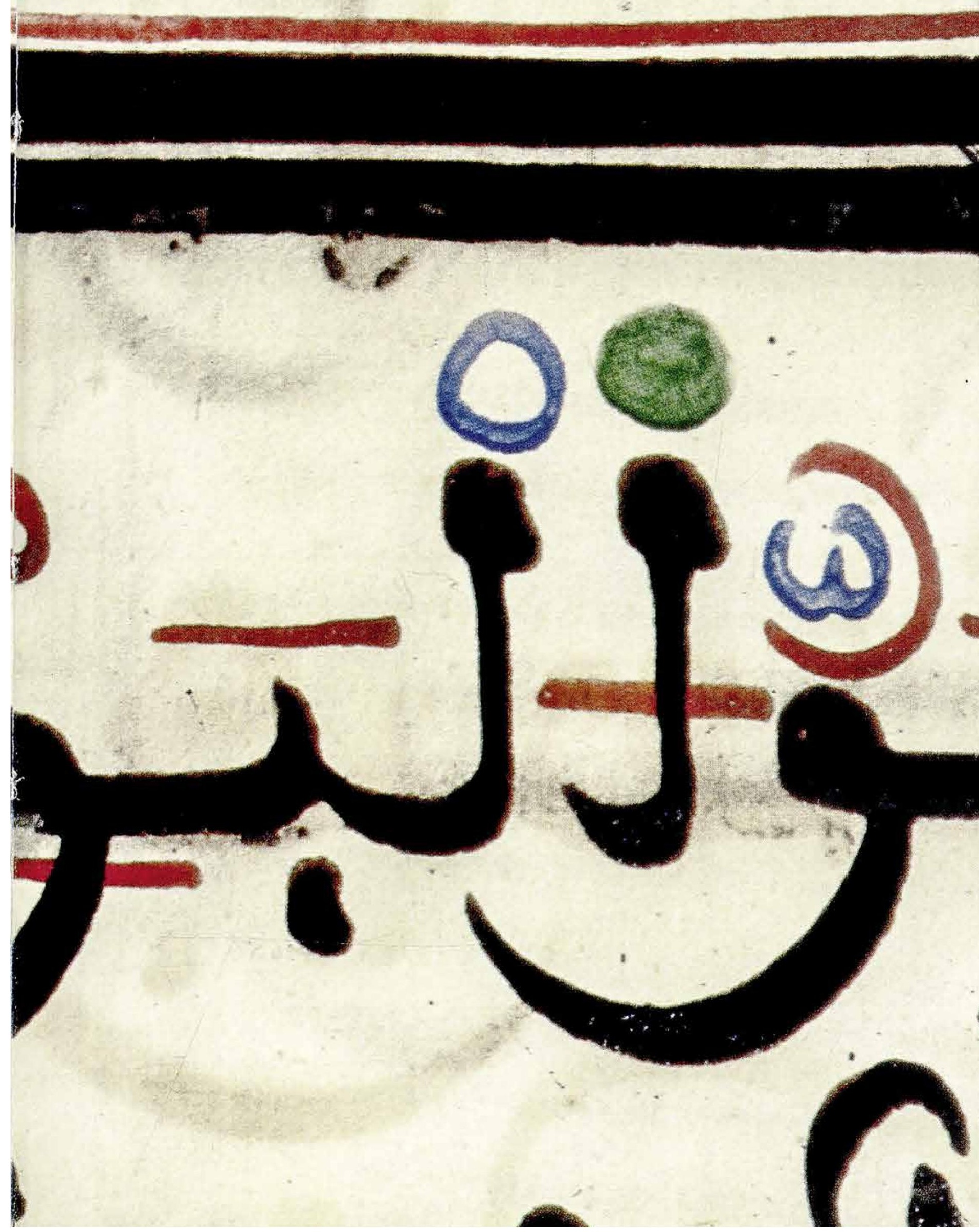




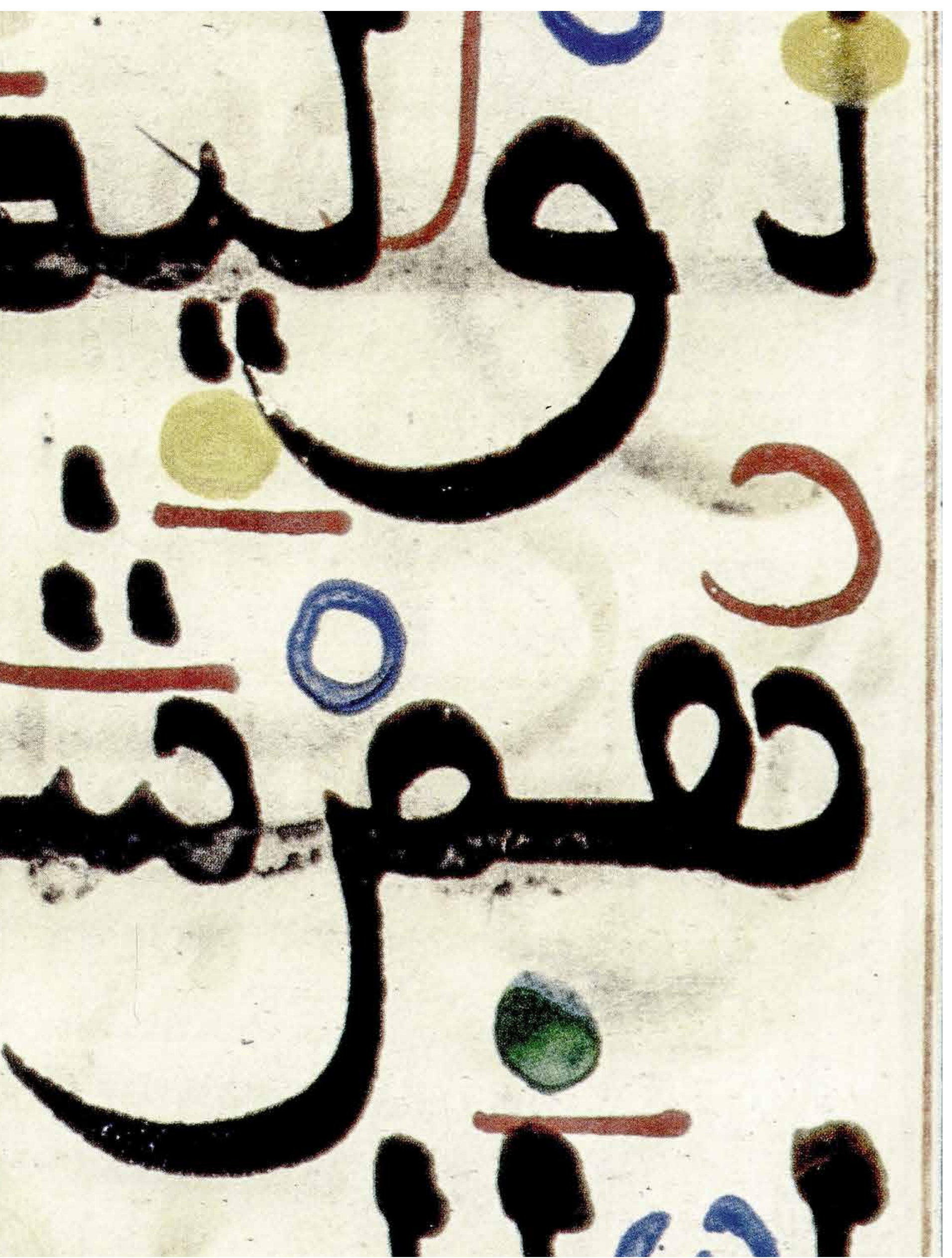


double page précédente

fig. 9

Manuscrit coranique du $x x^{e}$ siècle. Écriture du célèbre calligraphe marocain al-Qandoussi, mort en 1856. Les points diacritiques sont de couleurs différentes, in Sijelmassi 1987 : 204. marocanité authentique supposée activer une façon proprement locale d'entrer en relation avec Dieu. Enfin, dans cette médiation, les clercs institutionnels jouent un rôle central. On décèle une inquiétude à leur égard, révélatrice de l'effort consenti par le ministre pour les inciter à servir de caution dans la fabrication de nouvelles normes matérielles et sensorielles du Coran. L'importance accordée à leur jugement témoigne autant de la volonté du roi de les voir légitimer ses positions et sanctifier son action que de la priorité donnée à la forme des lettres intimement liée aux sons à émettre. La «bonne lettre» vise ainsi la «bonne récitation", c'est-à-dire la manière correcte de sonoriser le texte par le truchement de la voix.

\section{Réciter le Coran "à la marocaine"}

\section{Les sens du son}

«La bonne récitation", ou cantillation, dont le but principal est de régler la restitution exacte des textes sacrés de manière à prévenir les déviations dangereuses, a conduit à l'établissement du tajwîd comme branche des sciences coraniques. Le tajwîd - «la parure de la récitation» (Shiloah 1991: 91) - est un système consacré à la voix et à ses émissions, ainsi qu'à l'art de psalmodier selon les règles et les lois de la phonétique signalées par différentes couleurs dans les "Coran tajwîd", comme est appelée cette catégorie de livres didactiques (fig. 10). Les consonnes arabes étant inertes, cette science considère comme fondamental le traitement des voyelles qui les personnalisent sous différents aspects.

L'explication d'un clerc de la fondation est éloquente à ce propos: "Les musulmans se soucient de prononcer autant que possible le saint Coran suivant LA manière dont il a été révélé au prophète." Manière qui répond à des règles spécifiques conformes aux méthodes du tajwîd, propres aux récitations canoniques. II poursuit: «Il y a moussaaaaa, moussééé, moussi - récite-t-il en me dessinant un croissant représentant une échelle d'intensité allant du son « $a$ » au son « $i$ » - ça dépend de la récitation. » Les récitations ne sont pas des versions alternatives du texte mais renvoient à des variations mineures dans la vocalisation. II arrive cependant que les différentes prononciations d'un même mot entraînent des divergences d'interprétation. C'est pourquoi les clercs prêtent une attention minutieuse à la manière dont chaque lettre est sonorisée par la voix. Ce qui implique la plus grande précision, on l'a dit, quant à la forme des lettres et à l'emplacement des signes diacritiques. Ce souci d'interprétation nécessite en outre de régler les arrêts de la voix, distincts dans le Coran marocain et l'édition standard. Variation cruciale, car c'est de la manière de rythmer le texte que dépend la transmission du message, dans la mesure où s'arrêter à un endroit inadéquat de la phrase risquerait de produire un sens hérétique. Ainsi, les modalités délicates de déclinaison sonore d'une même voyelle ou d'un trait de ligature ont des propriétés cognitives.

\section{La récitation collective $d u$ hizb}

Toutefois, pour le directeur de la fondation, l'interprétation n'est pas l'enjeu essentiel du «bon » son à émettre. Ce qui importe est le déroulement de la récitation du hizb, qui doit répondre à un mouvement collectif: «ll faut que les Marocains puissent prier ensemble sans problème pendant le hizb", dit-il. Le hizb («partie» [désigne aussi le «parti politique»] - en référence aux 
soixante sections du Coran) désigne une récitation collective réalisée à la mosquée deux fois par jour, après les prières de l'aube et du coucher (de telle sorte que le Coran, composé de soixante hizb, soit intégralement récité dans le mois). Aux autres moments de la journée, les pratiquants écoutent en silence la voix de l'imam. Historiquement ancrée au Maroc (Fariji 2017), la récitation du hizb n'est quasiment pas pratiquée au MoyenOrient où des musulmans rigoristes la qualifient de bid'a («innovation »). Cette conception est de plus en plus répandue au Maroc, déplore le directeur de la fondation. II arrive même que des imams refusent la récitation du hizb dans leur mosquée alors même qu'elle fait partie d'un programme spécifique de leur formation mis en place par le ministère. L'enjeu est de taille puisque la pratique du hizb est indissociable de l'identité marocaine, selon le site du ministère.

D'une durée d'environ vingt minutes, cette récitation est dirigée par l'imam. «Tandis qu'il lit par cœur [c'est-à-dire récite], explique un jeune pratiquant, nous lisons le livre". Agenouillés en cercle autour de l'imam, les fidèles s'aident du livre pour ne pas commettre d'erreurs. "C'est pour ne rien oublier ", ajoute le pratiquant. Le livre occupe une place centrale dans le rituel initialement destiné à l'apprentissage par cœur du Coran suivant une diction correcte acquise grâce à la récitation répétée et collective. "Sauf que maintenant, rétorque le directeur de la fondation, les fidèles ne prient plus à partir du même livre, celui-là récite à l'orientale, l'autre à la marocaine: alors l'un s'arrête tandis que l'autre continue, puis il s'agenouille alors que l'autre reste debout car il n'a pas vu al-sajadât, il y a un désaccord. " Al-sajadât («prosternations") - signalées dans le texte par un médaillon - indique au fidèle quand effectuer le geste d'inclinaison au sol. Leur nombre varie suivant les éditions: onze dans le mushaf mohammadî, quatorze dans l'édition standard. Ainsi, les composantes matérielles du livre servent non seulement la mise en sons mais aussi la mise en gestes du Coran suivant des règles précises qui permettent de ne pas troubler la performance collective du rituel. C'est pourquoi, dans une volonté d'«homogénéisation ", déclare le directeur de la fondation, celle-ci a fait distribuer largement le mushaf mohammadî dans les mosquées pour que les fidèles prient à partir du même livre voué à régler les sons émis par la voix, les mouvements du corps et les sens associés à la prière. En établissant des normes d'écriture et d'ornementations, c'est bien une intonation, une sonorité et une gestuelle coraniques proprement marocaines qu'il s'agit de modéliser.

\section{Sons en circulation}

Pour ce faire, la monarchie s'est emparée d'autres médias que le livre, dont principalement la radio et la télévision. En plus des prières retransmises aux horaires habituels, des émissions consacrées à l'apprentissage de la récitation propre au mushaf mohammadî sont diffusées plusieurs fois par semaine. C'est donc autour du livre qu'est surtout axée la politique d'«homogénéisation " sonore: de sa confection à sa commercialisation.

Pour endiguer la circulation de copies du Coran non conformes aux règles établies par la fondation, celle-ci produit annuellement (au sein de l'imprimerie royale) environ un million d'exemplaires du mushaf mohammadî, voués à être distribués dans les mosquées. En outre, elle contrôle les exemplaires importés du Moyen-Orient grâce à l'édiction d'une nouvelle réglementation. Les distributeurs sont désormais contraints de remettre un formulaire 
précisant la récitation, le style calligraphique, le format, le type de couverture et les couleurs des modèles importés du Moyen-Orient où ils sont conçus et/ou imprimés. En amont de ce long protocole, ils fournissent aux imprimeurs moyen-orientaux «la feuille de route» établie par la fondation énonçant les règles à appliquer. «lls n'y comprenaient rien, raconte un distributeur. Mais ils étaient obligés de s'adapter. Avec ce qui se passe là-bas en ce moment, on est les seuls à acheter. " Seuls les producteurs égyptiens et libanais (à la fois éditeurs et imprimeurs) à la tête de structures capables de financer la conception de modèles inédits suivant les nouvelles règles du jeu commercial ont pu continuer à tirer profit du marché florissant du Coran au Maroc. Les logiques économiques ont donc favorisé - du moins pour un temps - la politique d'effacement de récitations jugées hétérodoxes et permis le développement d'un marché du Coran conforme aux exigences du Palais à l'échelle régionale.

\section{Différend signifiant: le cas du Coran tajwîd}

La fondation, sous la pression des clercs, a interdit les livres de Coran miniatures (à usages majoritairement talismaniques) et les exemplaires rangés dans des petits coffrets (généralement offerts à l'occasion des mariages et baptêmes), modèles également prohibés dans les pays rigoristes comme l'Arabie Saoudite. Les éditions avec du texte en couleurs pour mettre en évidence les noms de Dieu ou les règles de récitation ont aussi été interdites, alors qu'elles suscitent l'engouement des fidèles, de plus en plus nombreux à souhaiter apprendre le Coran «par eux-mêmes». Peu de temps après leur interdiction cautionnée par le ministre, l'intervention d'un éditeur marocain du quartier des Habous a semé le trouble. II raconte: «L'interdiction du Coran tajwîd, ah ça je n'ai pas accepté! Le producteur libanais allait devenir fou: le Coran tajwîd a été bloqué durant plus de six mois, il n'y en avait plus sur le marché, c'était un scandale, les libraires le vendaient à des prix élevés. Alors, j'ai demandé à la fondation qu'elle me prouve que cette interdiction venait du roi lui-même ou du gouvernement. » Devant le silence persistant du directeur de la fondation, l'éditeur a profité de l'inauguration du Salon international du livre et de l'édition de Casablanca en 2013 pour aller à la rencontre du chef du gouvernement: «Je me suis faufilé dans la foule, dit-il, et je lui ai offert un Coran tajwîd, il m'a remercié alors je lui ai demandé pourquoi ce Coran ne pouvait plus rentrer au Maroc. II a dit: "Mais c'est impossible, il y a une note spéciale concernant ce Coran!" Je lui ai juré que la fondation nous l'avait interdit. Puis je lui ai envoyé un dossier contenant tous mes échanges avec la fondation et dans la semaine j'ai obtenu l'autorisation d'importer le Coran tajwîd! " Le chef du gouvernement aurait exigé de l'institution la levée de l'interdiction. Décision à laquelle s'est plié le directeur à la demande du ministre qui a affronté l'opposition obstinée des clercs.

Ce contentieux met en lumière non seulement la hâte avec laquelle la nouvelle réglementation commerciale a été mise en place - le chef du gouvernement n'ayant pas été averti de la mesure à la veille du salon du livre - mais aussi la divergence d'opinion au sein du régime entre le ministre et le chef du gouvernement (également secrétaire du Parti de la justice et du développement) sur l'attitude à adopter vis-à-vis du Coran tajwîd ou plutôt sur la manière dont le Coran doit être mémorisé et transmis. Si, pour le roi et ses conseillers, les normes définies par les clercs institutionnels sont seules valables, aux yeux du chef du gouvernement, le Coran doit pouvoir s'apprendre de manière 

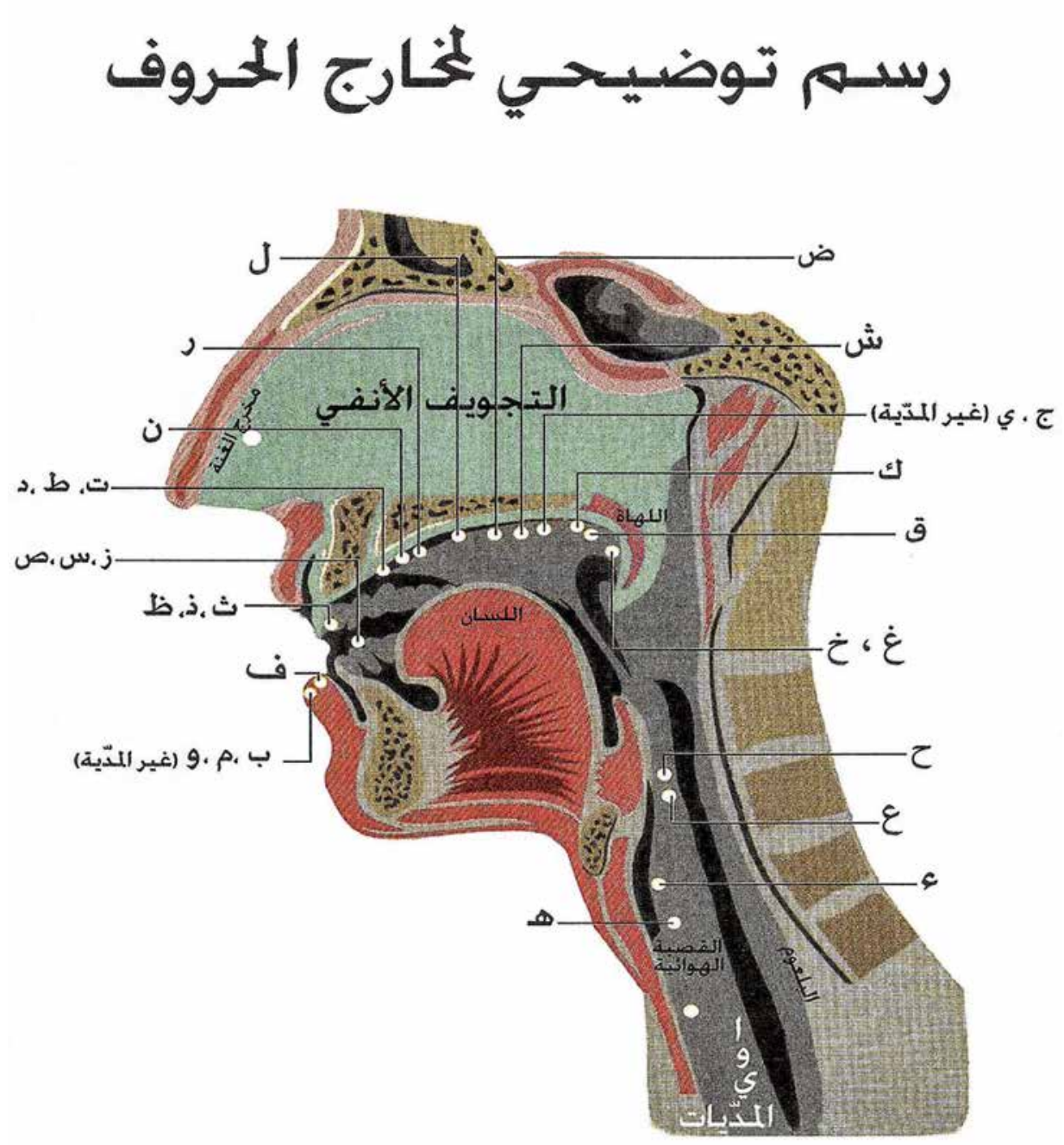

autonome et directe, sans la médiation imposée de l'État. Cet épisode illustre également le souci du monarque de ne pas se montrer absolutiste à un moment où il doit faire preuve d'ouverture et de souplesse à l'égard du parti islamiste au pouvoir.

Ainsi, à la dimension fortement collective et socialisée des usages du Coran qui trouve son expression la plus emblématique dans le rituel du hizb, où la mise en sons et en gestes est supposée fabriquer une identité religieuse marocaine homogène, s'ajoute un autre enjeu, intimement lié au premier: apprendre par cœur le Coran.

\section{Les sens de l'apprentissage}

\section{Avoir le Coran sur les lèvres}

Selon la méthode traditionnelle encore répandue au Maroc, l'enfant, une fois qu'il maîtrise l'alphabet, apprend le Coran en reproduisant à partir d'un calque les versets écrits par son maître. Sa planche en bois (fig. 11 et 12) maintenue à la verticale, l'enfant répète le texte à voix haute à la suite de son professeur tout en le copiant. Plus ancrée au Maroc qu'ailleurs dans le monde islamique (Eickelman 1978; Messick 1996), la mémorisation (hifz) ainsi effectuée dépasse largement l'apprentissage du texte: elle vise la fig. 10

Dessin représentant l'articulation des lettres à la fin d'un Coran tajwîd, Dar al-Maarifah, Damas, 2003: 64. 
16. Notons à ce propos que la poésie est une pratique mnémotechnique profondément ancrée dans l'apprentissage du Coran. Au Maroc, comme ailleurs, elle inculque le rythme jugé fondamental dans la récitation et la compréhension du texte (Geertz 1983).

17. Un article en cours de préparation examine les ressorts cognitifs de l'écriture iconique du livre saint dans le cadre de l'apprentissage du Coran par des clercs auto-proclamés à Casablanca.

\section{ci-contre}

fig. 11

Romain Simenel,

Récitation du Coran dans une école du Sud marocain (C) Romain Simenel, 2010. transformation de l'apprenant au sens fort du terme. L'enfant doit littéralement posséder le Coran (Eickelman 1978), l'incorporer (Ware 2014), comme le laissent entendre les expressions courantes au Maroc: «avoir le Coran sur les lèvres" (Eickelman 1986: 48) ou "porter le Coran dans son Cœur" (Fortier 1998: 218). Cette véritable mise en corps de la Parole de Dieu doit prodiguer valeur morale et éthique à l'élève dont le corps physique imprégné par la sacralité du Verbe est vu comme l'agent transformateur de l'esprit.

Au Maroc, comme dans d'autres pays musulmans, l'apprentissage par cœur du Coran repose sur des marqueurs mnémoniques (Eickelman 1978) qui s'apparentent aux procédés développés dans la Grèce et la Rome antiques pour faciliter la mémorisation des textes, sacrés ou non, à travers «lieux» et «images» (Yates 1986 [1975]). Ici, ce sont davantage l'intonation, le rythme ${ }^{16}$ ou encore l'association visuelle entre la forme des lettres et la morphologie des animaux (Simenel 2015) qui se combinent dans un enseignement synesthésique visant à soutenir une incorporation exacte et optimale du Coran. On comprend mieux que les propriétés physiques du texte qui configurent les manières spécifiques de le voir, de l'entendre et de le réciter, représentent un enjeu majeur. Notons à cet égard que les "grands récitants", les hazzâb - désignant ceux qui connaissent le Coran par cœur et le récitent - emploient généralement une seule édition. C'est la méthode recommandée par les maîtres à leurs élèves incités à recourir au livre comme à un objet mnémotechnique. Beaucoup de fidèles accordent un intérêt particulier, par exemple, à la composition graphique, préférant que les pages se terminent à la fin des versets plutôt qu'en leur milieu (Cohen 2012). Au cours de leur apprentissage, la page est associée à une image (autre signification donnée au mot rasm, "calligraphie") qu'il s'agit de fixer visuellement dans sa mémoire pour retenir les sourates. Sur la page, tout compte, disent les récitants: le style d'écriture, la mise en pages et les décorations ${ }^{17}$.

Ainsi les médaillons, les rosettes et les titres de sourate ne remplissent pas seulement une fonction décorative, symbolique et spirituelle (Lings 1976). En signalant la fin d'un verset ou le début d'une sourate, ils en facilitent la mémorisation. Plus que de rappeler l'ordre des versets aux pratiquants, les médaillons signalent, on l'a dit, une action physique: la prosternation requise à la fin de certains versets. Dans cette perspective, les ornementations qui ont pour «fonction d'attribuer de la saillance mnémonique aux variations » du texte (Severi 2009: 476) engagent les fidèles sur un mode plus participatif que contemplatif dans leur relation à Dieu. Comme dans l'art roman au Moyen Âge, «le traitement différencié et la distribution calculée de ces dispositifs ornementaux font qu'ils servent à hiérarchiser ce qu'ils affectent, mais aussi à en accompagner (au sens musical), à en orienter et à en rythmer l'investissement rituel. [...] En cela, l'ornemental, à défaut d'une signification discursive et symbolique explicite, a au moins un sens expressif (intensif, qualificatif) qui est éprouvé, voire même agi » (Bonne et al. 2010: 29).

Ainsi, le livre du Coran serait en lui-même une mnémonique, "une technologie de la mémoire", au sens où Mary Carruthers (1990) entend cette notion, soit une grammaire visuelle d'images servant d'amorce mnésique au texte, en particulier dans la Bible de l'Europe médiévale. Plus encore, Mary Carruthers montre comment la mémoire ainsi disciplinée, entraînée et éduquée consistait en une transformation de la connaissance en expérience et permettait à une œuvre de s'institutionnaliser dans le langage et l'imaginaire des individus. Serait-ce cette technologie de la mémoire que 


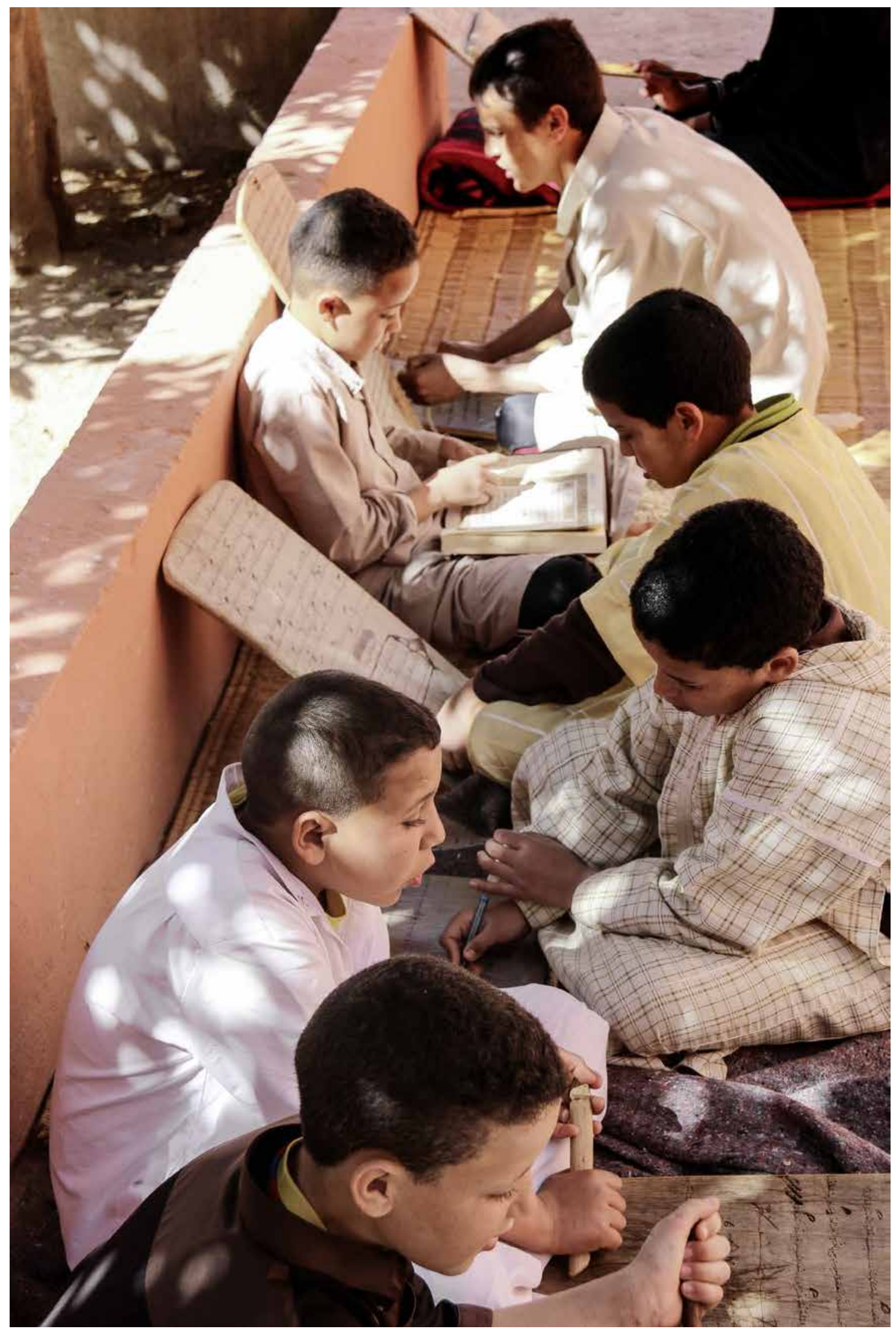




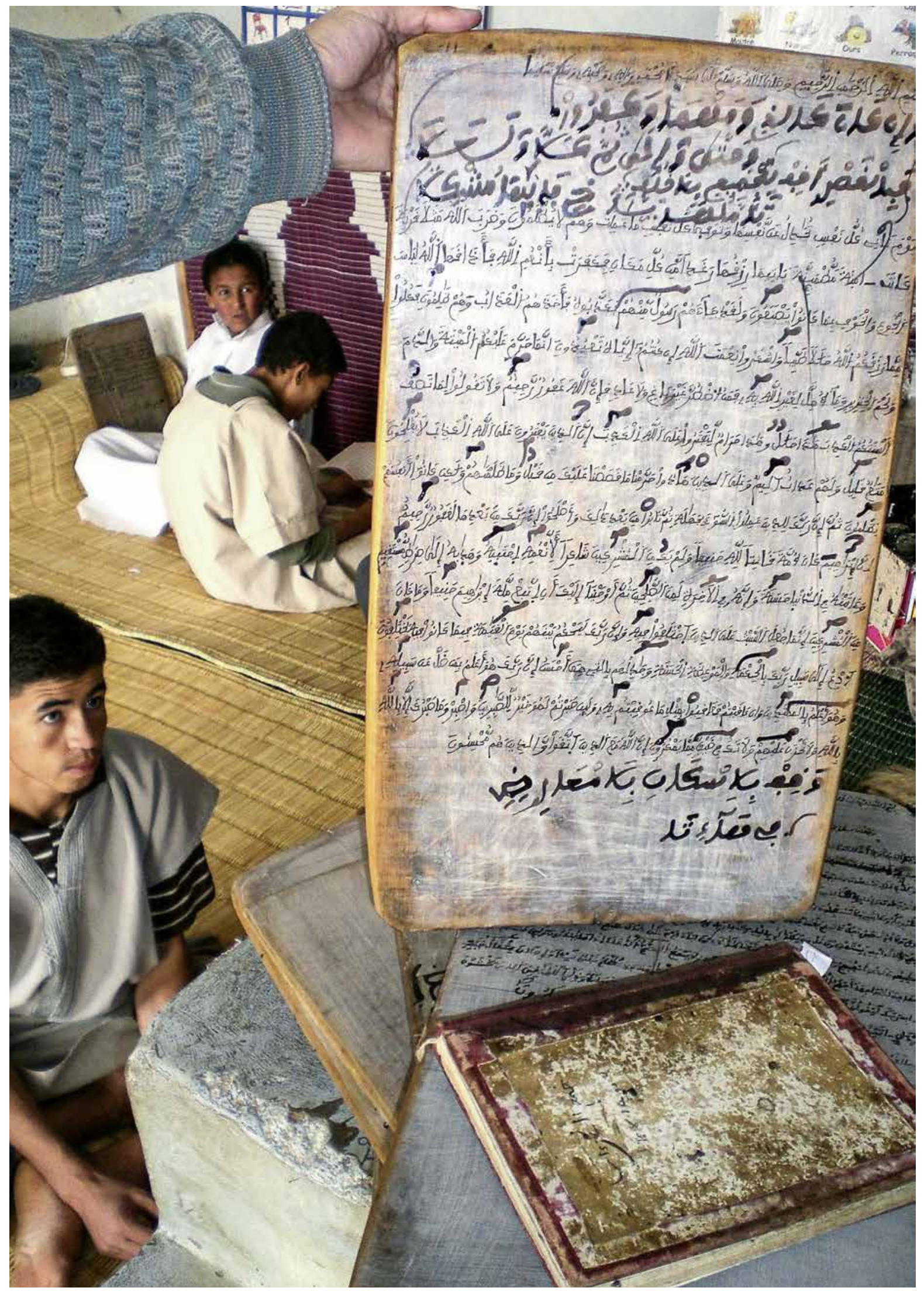


le Palais cherche à modéliser à travers la reconfiguration matérielle du Livre? Serait-ce en un mot la manière d'incorporer le texte, de le faire sien qui doit être rendue marocaine, homogène et étatisée? Discipliner les sens, définir et contrôler les façons de voir et d'entendre le Coran, sont en effet placés au cœur d'une politique dont les deux principaux espaces d'intervention sont les mosquées et les écoles.

\section{Discipliner les sens dans les mosquées...}

Chaque année depuis 2012, des centaines de milliers d'exemplaires du Coran marocain sont placés dans les mosquées afin de contrecarrer une pratique de plus en plus courante: les donations de livres saints aux lieux de culte. Les fidèles qui souhaitent «faire sadaqa» («aumône») les offrent par dizaines, par centaines, voire par milliers pour, disent-ils, se «sentir proche[s] de Dieu » (Cohen 2010). Généralement, c'est l'imam de la mosquée à laquelle ils souhaitent adresser le don qui indique l'édition à acheter. Cependant, ils peuvent aussi décider du modèle qu'ils veulent acquérir et distribuer. De sorte qu'on trouve une variété de versions, comme le soulignait plus haut le directeur de la fondation. Jusqu'à une période récente, l'approvisionnement des mosquées en équipements et objets liturgiques était libre, tout comme leur construction. Selon le recensement du ministère des Affaires islamiques réalisé en 2006 , près de $80 \%$ des mosquées ont été fondées par un bienfaiteur. Depuis le nouveau décret voté en 2008, le mécène doit désormais demander l'autorisation avant d'ouvrir une mosquée et faire approuver le recrutement du personnel religieux qu'il est chargé de rémunérer. Ainsi, la gestion des mosquées, qui relevait auparavant d'un fonctionnement informel, est aujourd'hui placée au cœur d'une «bureaucratie du croire » (Tozy 2013) appelée à rationaliser un système reposant jusqu'alors sur des rapports interpersonnels et hérités. Cette gestion s'apparente au dispositif de contrôle mis en place en Arabie Saoudite qui assure une veille quotidienne pour «imposer l'habitus wahabbite» (Mouline 2017: 67). Dans ce pays où l'orthodoxie et l'orthopraxie de référence sont au cœur des mécanismes de légitimation politique (Mouline 2011), l'effectuation de la prière, socle de l'islamité de l'individu et du groupe et de la cohésion communautaire, tient une place centrale dans la construction de la religion d'État. Le Maroc semble emprunter la même voie.

Depuis une période récente en effet, les délégations régionales procèdent à des contrôles réguliers, attentives en particulier à la manière dont l'imam récite le Coran et corrige les erreurs commises par les fidèles. Le, «guide de l'imam », récemment conçu par le ministère des Affaires islamiques, stipule les normes de l'appel à la prière se devant d'être proclamée «à la manière marocaine [...] sur un ton naturel et préservé de toute imitation étrangère». Aucune loi restrictive n'est appliquée, toutefois les contrôleurs restent vigilants: "S'il y a des Corans qui ne sont pas en règle, soit parce qu'ils sont déchirés, soit parce qu'ils reprennent la récitation orientale, les contrôleurs le déclarent au délégué, qui récupèrent les Corans qu'on remplace ensuite nous-mêmes par le mushaf mohammadî. Le tout, c'est que les fidèles aient un Coran avec lequel prier», explique le chargé de gestion du département des mosquées. Procéder par substitution est la stratégie adoptée pour faire du mushaf mohammadî la version dominante. «On doit corriger doucement et ne pas entrer en conflit », poursuit l'employé.

\section{ci-contre}

fig. 12

Romain Simenel,

Un élève et sa planche

coranique dans une école

du Sud marocain

(C) Romain Simenel, 2010. 


\section{ci-contre}

fig. 13

Page d'un manuel reproduisant les gestes à adopter durant les prières, Dar Attakafa, Casablanca, 2003: 46.
Le principal enjeu de cette "correction" est, on l'a dit, la récitation collective du hizb destinée à soutenir la mémorisation du Coran suivant la méthode de récitation conforme. À Rabat comme à Casablanca, les cours d'alphabétisation et d'instruction islamiques, délivrés dans les mosquées auxquels assistent de plus en plus d'hommes et de femmes généralement éduqués, représentent un autre enjeu majeur (El Ayadi et al. 2006). Les jeunes, eux, participent rarement à ces enseignements, préférant souvent lire et écouter le Coran sur leur téléphone portable (ibid.), comme de nombreux fidèles plus âgés. Des applications de téléphone mobile sont maintenant disponibles, qui mettent à disposition des usagers plusieurs récitations ainsi que l'explication de certains termes. De plus, contrairement aux personnes ayant appris le Coran à l'école coranique, beaucoup de jeunes fidèles préfèrent acheter le Coran conforme à la récitation orientale à laquelle ils se sont familiarisés à travers les chaînes de radio et de télévision égyptiennes qui dominent les ondes marocaines. C'est aussi le modèle reproduit dans le style d'écriture qu'ils sont habitués à lire dans les journaux, les livres et les manuels scolaires.

Le directeur de la fondation, convaincu de la politique menée par le ministère, n'est pourtant pas inquiet: «On apprend le Coran à la mosquée. Si de plus en plus de jeunes écoutent le Coran à la télévision ou à la radio, ça ne pose pas problème car généralement quand ils vont à la mosquée, ils trouvent l'imam qui récite à la marocaine. " Tout le monde n'est pas de cet avis, en particulier les clercs de la fondation qui se lamentent: "Aux concours de psalmodie du Coran organisés au Maroc et dans le monde, on a commencé à remarquer que nos jeunes récitaient beaucoup à l'orientale. " Devant le déclin de la récitation marocaine et le recul de la calligraphie maghribî dont l'emploi permettrait, selon les clercs, de protéger le texte coranique de toute falsification introduite par les nouveaux moyens de production en calligraphie naskhî (écriture orientale), la fondation a souhaité intervenir au plus près des enfants: dans les écoles.

\section{... et les écoles}

Écoles coraniques, cours d'instruction islamique donnés par un professeur bénévole dans son appartement, classes d'alphabétisation destinées aux adultes au sein d'associations caritatives, jardins d'enfants, etc.: I'offre d'instruction religieuse s'est diversifiée depuis le milieu des années 2000. Dans ces établissements majoritairement privés, le tableau noir, le cahier et les petits livres du Coran (reprenant les sourates les plus courtes que les enfants apprennent en premier) remplacent de plus en plus la planche en bois. À la relation directe et interpersonnelle maître/élève se substituent des dispositifs cognitifs où l'imprimé joue un rôle central. Or, jusqu'à récemment, les professeurs d'écoles privées (ou semi-privées lorsqu'elles sont subventionnées par l'État) étaient totalement libres d'employer les supports pédagogiques de leur choix. Beaucoup enseignaient à partir de manuels scolaires conformes à la calligraphie et à la récitation dites orientales: "C'est plus facile pour les élèves", déclare un professeur dans une école élémentaire à Casablanca. Ces manuels reproduisent en images les gestes à adopter durant les prières, suivant la pratique couramment admise au Moyen-Orient où les fidèles se prosternent les bras le long du corps et non croisés sur la poitrine comme on le voit souventau Maroc (fig. 13). 


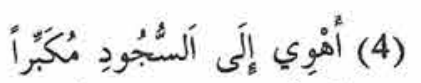

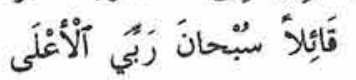

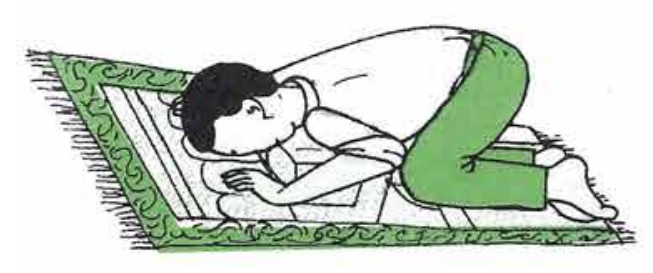

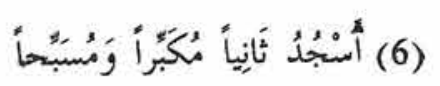
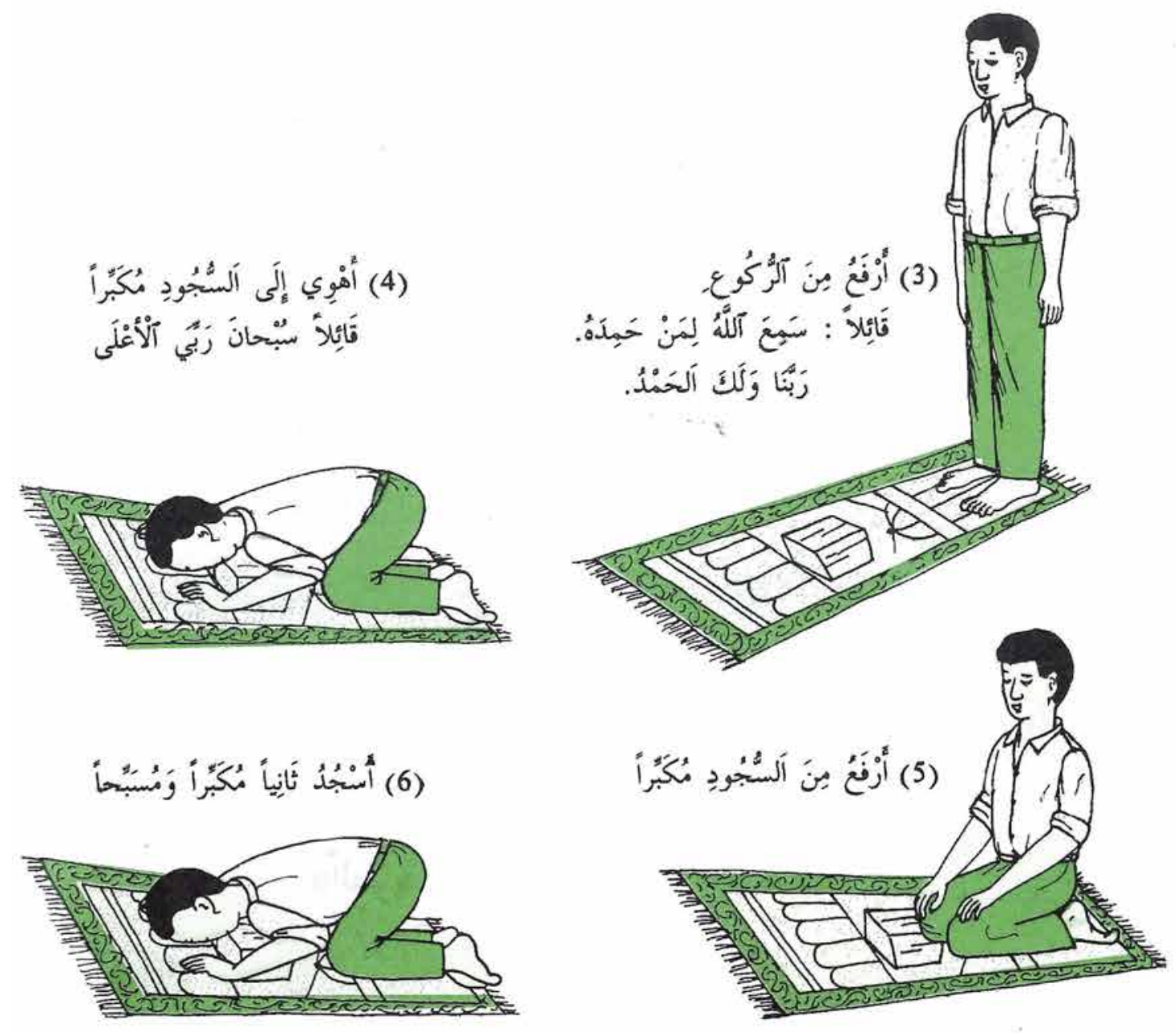

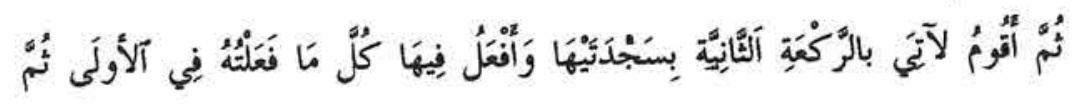

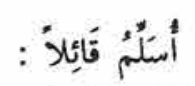

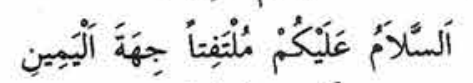

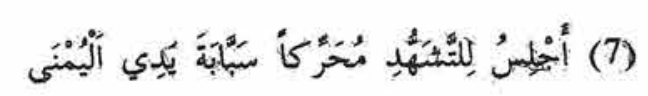
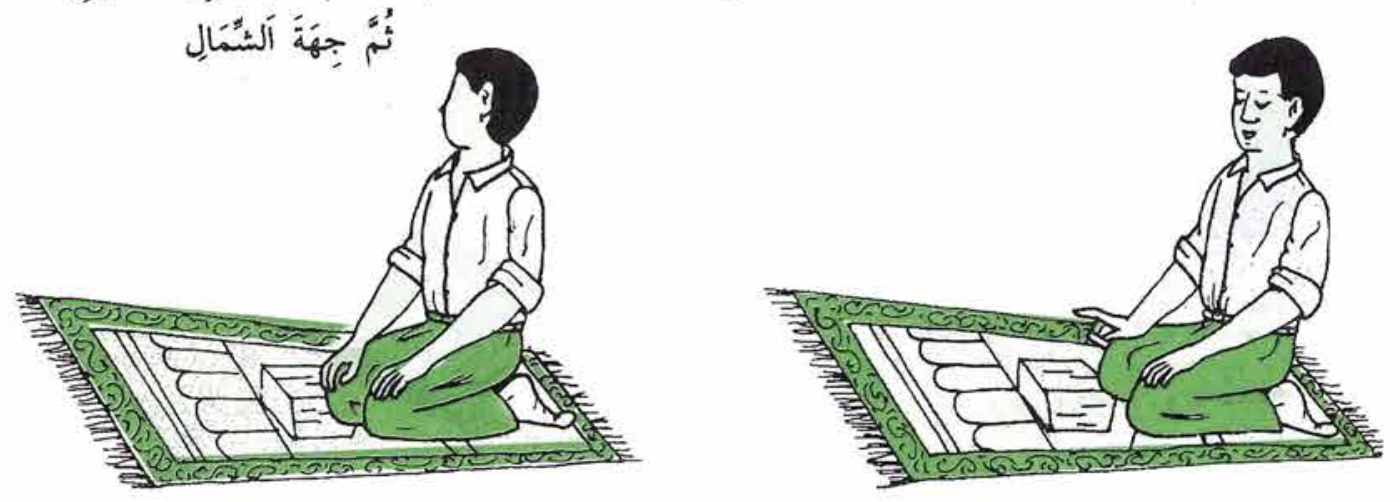
18. Dans le cadre de la réforme, le ministère des Affaires islamiques, à présent composé de quatre départements - affaires islamiques, mosquées, enseignement traditionnel, prédication a vu son effectif quasiment quadrupler (Tozy 2013).
En réponse à ces pratiques considérées comme des infractions à la norme, le département de «l'enseignement traditionnel » du ministère a pris ses dispositions ${ }^{\mathbf{1 8}}$. «La seule doctrine qui doit être suivie pour les manuels scolaires est celle du mushaf mohammadî», stipule la feuille de route déjà évoquée. Toutefois, dans ce domaine, l'État n'a pas non plus souhaité légiférer, redoutant d'affronter les associations de défense des droits de l'homme. Pour veiller au respect des nouvelles règles, des contrôleurs du département se rendent fréquemment dans les établissements afin de vérifier aussi bien les méthodes d'enseignement que les supports d'apprentissage employés. Néanmoins, de nombreuses «maisons du Coran» (dar el qur'an), telles que les appellent communément leurs mécènes, échappent aux contrôles: «ll s'en construit de nouvelles tous les jours», dit un employé du ministère. Une fois de plus, le moyen de pression utilisé est le levier économique: les écoles déviantes cessent de recevoir les subventions publiques.

Parallèlement aux écoles privées, les structures d'enseignement étatiques font également l'objet d'une attention particulière. Un manuel scolaire d'apprentissage islamique préparé par le ministère de l'Éducation nationale en concertation avec la fondation, intègre des extraits du mushaf mohammadî à côté de passages tirés de l'édition standard. Introduit au programme scolaire de l'école élémentaire depuis 2016, ce nouveau manuel vise à habituer les jeunes générations à l'apparence physique du mushaf mohammadî sans rompre brutalement avec leurs habitudes d'écriture et de récitation. Selon le directeur du département, la réforme nécessite une transition souple: «On ne peut pas imposer la calligraphie maroco-andalouse à quelqu'un qui n'écrit même pas bien; la plupart des professeurs écrivent comme des MoyenOrientaux. Donc on essaie de les habituer, comme ça l'élève pourra au moins lire le Coran chaque fois qu'il ira à la mosquée. » Ces observations renforcent l'idée qu'il y aurait pour le Palais un pouvoir effectif de la matérialité du livre (et du manuel) sur la formation du sujet religieux.

\section{De la praxis à l'idéologie: le parti pris de l'écoute}

En dehors du livre, les membres du ministère attribuent des propriétés efficaces aux sons, établissant un lien étroit entre la manière de réciter le Coran et l'influence d'une théologie et d'une doctrine. Le directeur de la fondation l'exprime de manière éloquente: «À travers la récitation orientale on apprend aux enfants à sortir du malékisme. Par exemple, quand on dit à un enfant: "On ne lit pas de cette manière, on s'arrête là et pas là", petit à petit ça touche à autre chose, c'est le dogme sans être le dogme. Voilà pourquoi notre but n'est pas de répandre le mushaf mohammadî, c'est autre chose: c'est politique, c'est l'extrémisme. Dans presque toutes les maisons du Coran au Maroc c'est la récitation orientale. Ils attirent les orphelins, ils leur donnent de quoi manger et puis ils leur apprennent le dogme wahabbite. Après, ils sont envoyés en Arabie Saoudite et puis ils reviennent comme des bombes! » Ainsi, entendre ne serait pas neutre. Ce serait un acte lié à une idéologie spécifique incorporée par les fidèles au même titre que les sons qui la caractérisent.

La nouvelle gestion des mosquées et des écoles donne à voir la relation clairement établie par l'institution entre la praxis, la doctrine et l'extrémisme: allonger la lettre à tel endroit, s'arrêter au bon moment, se prosterner de telle manière, etc. Ce sont à ces gestes-là, corporels et sensoriels, qu'on donne de l'importance, à l'origine de la création d'une édition marocaine du livre saint vouée à discipliner la mise en actes du Coran. Comme si l'État 
supposait que la protestation des fidèles décrits comme islamistes passait avant tout par l'usage ordinaire d'un objet placé au cœur de leur quotidienneté et par l'imprégnation dans les corps de gestes et de sens spécifiques. Ce sont ces manières de se mouvoir et d'être connecté à la parole de Dieu, supposément activées par les composantes matérielles du livre, qu'il entend façonner ${ }^{19}$.

\section{Conclusion}

Le mushaf mohammadî est conçu comme l'outil d'une homogénéisation des manières de voir et d'entendre le Coran, l'objet devant susciter une expérience sensible commune et produire un mode de connaissance susceptible de transformer concrètement les subjectivités religieuses selon une orthodoxie stricte. À travers l'élaboration d'une «formation esthétique 20 » voulue hégémonique, le monarque cherche à gouverner l'engagement sensible des fidèles avec le Coran, pensé ici comme un dispositif graphique, mnémonique et cognitif ayant son efficacité propre. Lire le mushaf mohammadî constituerait une forme d'allégeance au roi, au point que rejeter «son Coran » reviendrait à s'écarter d'Allah.

Par la reconfiguration matérielle du livre et la fabrication d'un rapport plus sensoriel qu'intellectuel au texte, il s'agit de réorienter les usages visuels et sonores du Coran afin qu'ils évoluent dans un champ de pratiques délimité, circonscrit et facilement maîtrisable ${ }^{\mathbf{2 1}}$. L'enjeu d'un tel projet est l'affirmation de l'autorité religieuse du monarque commandeur des croyants et la formation d'une subjectivité religieuse identitaire qui doit jouer un rôle rassembleur. Celle-ci passe aussi bien par la construction de certaines normes sensorielles que par l'effacement ou encore l'anaesthisation («anesthésie ») [Verrips 2006] d'autres normes. En réaction à la standardisation du croire résultant d'une déterritorialisation des produits religieux symptomatique d'une séparation entre culture et religion (Roy 2008), la monarchie travaille aujourd'hui à la (re)connexion des marqueurs religieux et culturel. À ce stade de l'analyse, une question principale reste en suspens: cette politique à peine entamée parviendra-t-elle à «faire marcher » les croyants (Certeau 1982: 57-58) ${ }^{22}$ ? Affaire à suivre dans une étude à venir des usages concrets du mushaf mohammadî par des fidèles ouverts à un monde globalisé de pratiques et probablement plus ingénieux et incrédules que cette politique ne pourrait le laisser croire.

CNRS/LESC anouk.cohen@wanadoo.fr
19. À cette fin, le mushaf mohammadîet ses traductions française et anglaise sont aussi distribuées en Europe et en Afrique de l'ouest.

20. Elle désigne selon Birgit Meyer ce qui «saisit bien l'impact de la formation d'une esthétique partagée à travers laquelle les sujets sont façonnés par la modélisation de leurs sens, les expériences induites, le moulage des corps et la fabrication de signification matérialisant cette esthétique » (Meyer 2009: 7, traduction de l'auteur).

21. Sur ce thème en contexte juif, voir l'étude menée par Jeremy Stolow aux États-Unis (2010).

22. "Toute autorité se fonde [...] sur le réel qu'elle est supposée déclarer. C'est toujours au nom d'un réel qu'on "fait marcher" des croyants et qu'on les produit. " L'extrait de ce chapitre de De Certeau est cité par Jean-Pierre Warnier lors d'une intervention (décembre 2013) où il analyse: «Certeau se propose de déconstruire la manière dont l'institution historienne, les enseignants, les éditeurs, etc. produisent le lecteur en tant que croyant de ces événements rendus réels au présent par les récits savants qui en sont faits. Cela "fait marcher" le lecteur. Celui-ci marche - pas toujours, mais le plus souvent. C'est ainsi que l'histoire se déploie entre science et fiction. » 


\section{Albin, Michel}

2005 «Printing of the Qur'an", in Jane Dammen McAuliffe (dir.), Encyclopedia of the Qur'an. Leiden, Brill.

\section{Asad, Talal}

2001 "Reading a Modern Classic: W. C. Smith's, The meaning and End of Religion ", History of Religions 40 (3) : 205-222.

\section{Bloch, Marc}

\section{3 [1924]}

Les Rois thaumaturges, étude sur le caractère surnaturel attribué à la puissance royale, particulièrement en France et en Angleterre.

Paris, Gallimard.

\section{Bonne, Jean-Claude, Denoyelle, Martine, Michel, Christian, Nouvel-Kammerer, Odile et Coquery, Emmanuel}

2010 " $Y$ a-t-il une lecture symbolique de l'ornement? ", Perspective 1 (2) : 27-42.

\section{Carruthers, Mary}

1990 The Book of Memory: a Study of Memory in medieval Culture. Cambridge, Cambridge University Press.

\section{Certeau (de), Michel}

1982 La Fable mystique: $x v I^{e}-x v I^{e}$ siècles. Paris, Gallimard.

1990 L'Invention du quotidien: arts de faire, t. I. Paris, Gallimard.

\section{Cohen, Anouk}

2010 "Le livre du Coran à Casablanca et à Rabat ", Archives des sciences sociales des religions 150: 175-195

2012 "Le Coran et ses multiples formes (Casablanca, Maroc) ", Terrain 59: 70-87.

2016 "Éditer la Révélation. Le Coran dans le Maroc contemporain ", Genèses 105: 57-75.

\section{Combs-Schilling, Elaine}

1989 Sacred performances: Islam, Sexuality and Sacrifice. New York, Columbia University Press.

\section{Eickelman, Dale}

1978 "The Art of Memory: Islamic Education and its Social Reproduction ", Comparative Studies in Society and History 20 (4) : 485-516.

1986 Knowledge and Power in Morocco: the Education of a Twentieth Century Notable. Princeton, Princeton University Press.

\section{El Ayadi, Mohammed, Rachik, Hassan et Tozy, Mohamed}

2007 L'Islam au quotidien: enquête sur les pratiques religieuses au Maroc. Casablanca, Prologues.

\section{Farag, Iman}

$\mathbf{2 0 0 9}$ «Querelle de minarets en Égypte. Le débat public sur l'appel à la prière ",

Revue des mondes musulmans et de la Méditerranée 125: 47-66.

\section{Fariji, Anis}

2017 "Le recto tono dans la récitation collective du Coran dite "lecture du hizb" : une image sonore d'un espace sacré étendu ", Hespéris-Tamuda, L II (2) : 179-200.

\section{Fawzi, Abdulrazak}

1990 "The Kingdom of the Book: the History of Printing as an Agency of Change in Morocco between 1865 and 1912 ", thèse d'histoire, université de Boston.

\section{Fortier, Corinne}

1998 «Le corps comme mémoire: du giron maternel à la férule du maître coranique ", Journal des africanistes 68 (1): 197-224.

\section{Foucault, Michel}

1975 "Pouvoir et corps", in Daniel Defert et François Ewald (éd.), Dits et écrits : 1970-1975, t. II. Paris, Gallimard, 1994

\section{Grabar, Oleg}

1992 The Mediation of Ornament. Princeton, Princeton University Press.

\section{Geertz, Clifford}

1971 Islam observed: Religious Development in Morocco and Indonesia. Chicago, University of Chicago Press.
1983 Local Knowledge: Further Essays in Interpretative Anthropology. New York, Basic Books.

\section{Hirschkind, Charles}

2005 "The Qur'an and the Media ", in Jane Dammen McAuliffe (dir.),

Encyclopaedia of the Qur'an. Leiden, Brill.

2006 The Ethical Soundscape: Cassette Sermons and Islamic Counterpublics. New York, Columbia University Press.

\section{Kantorowicz, Ernst}

1989 Les Deux Corps du roi: essai sur la théologie politique au Moyen Âge. Paris, Gallimard.

\section{Latour, Bruno}

2006 Changer de société, refaire de la sociologie, trad. de l'anglais par Nicolas Guilhot.

Paris, La Découverte.

\section{Lemonnier, Pierre}

2004 "Mythiques chaînes opératoires ", Techniques \& Culture 43-44.

\section{Lings, Martin}

1976 The Quranic Art of Calligraphy and Illumination. Londres,

World of islam festival.

\section{Mahmood, Saba}

2009 Politique de la piété:

le féminisme à l'épreuve

du renouveau islamique.

Paris, La Découverte.

\section{Messick, Brinkley}

1993 The Calligraphic State: Textual Domination and History in a Muslim Society. Berkeley, University of California Press.

\section{Meyer, Birgit}

2009 Aesthetic Formations: Media, Religion and the Senses.

New York, Palgrave Macmillan.

\section{Moors, Annelies}

2012 «Popularizing Islam: Muslims and Materiality - Introduction ", Material Religion 8: 272-279.

\section{Mouline, Nabil}

$\mathbf{2 0 0 9}$ Le Califat imaginaire d'Ahmad al-Mansûr: pouvoir et diplomatie au Maroc au $x v I^{\circ}$ siècle. Paris, PUF.
2011 Les Clercs de l'islam: autorité religieuse et pouvoir politique en Arabie Saoudite (xvIII ${ }^{e}-x x I^{e}$ siècles). Paris, PUF.

\section{$\mathbf{2 0 1 7}$ «Les prétentions}

hégémoniques du wahabbisme ", in Sabrina Mervin et Nabil Mouline (dir.), Islams politiques: courants, doctrines et idéologies.

Paris, CNRS Éditions: $37-70$.

\section{Robinson, Francis}

1993 "Technology and Religious Change: Islam and the Impact of Print ", Modern Asian Studies 27 (1): 229-251.

\section{Roy, Olivier}

$\mathbf{2 0 0 8}$ La Sainte Ignorance: le temps de la religion sans culture. Paris, Seuil.

\section{Severi, Carlo}

2009 "L'univers des arts de la mémoire: anthropologie d'un artefact mental ", Annales 2 (4): 463-497.

\section{Shiloah, Amnon}

1991 «La voix et les techniques vocales chez les Arabes ", Cahiers d'ethnomusicologie 4 : 85-101.

\section{Sijelmassi, Mohamed}

1987 Enluminures des manuscrits royaux au Maroc. Rabat, ACR Editions.

\section{Simenel, Romain}

2015 «Figuration, Prosody and Poetry: how Text comes to Mind in Southern Morocco ", intervention au $114^{\circ}$ congrès annuel de l'American Association Anthropology, Denver.

\section{Stolow, Jeremy}

2010 Orthodox by Design: Judaism, Print Politics and the Artscroll Revolution. Oakland, University of California Press.

\section{Sijelmassi, Mohamed}

1987, Enluminures des manuscrits royaux au Maroc. Rabat, ACR Editions.

\section{Tozy, Mohamed}

1999 Monarchie et islam politique au Maroc. Paris, Presses de Sciences Po. 
2009 "L'évolution du champ religieux marocain au défi de la mondialisation ",

Revue internationale de politique comparée 16 (1) : 63-81.

2013 "Des oulémas frondeurs à la bureaucratie du "croire". Les péripéties d'une restructuration annoncée du champ religieux au Maroc ", in Béatrice Hibou (dir.), La Bureaucratisation néolibérale. Paris, La Découverte: 129-154.

\section{Troupeau, Gérard}

1997 «les principes de l'écriture arabe ", in Annie Berthier et Anne Zali (dir.), L'Aventure des écritures: naissances, catalogue d'exposition (Paris, Bibliothèque nationale de France). Paris, BnF. Disponible sur: http://classes.bnf.fr/ecritures/arret/ lesecritures/arabe/02.htm (consulté le 19 février 2017).

\section{Verrips, Jojada}

2006 "Aisthesis and An-aesthesia", Ethnologia Europea 35 : 27-33.

\section{Ware, Rudolph}

2014 The Walking Qur'an: Islamic Education, Embodied Knowledge and History in West Africa.

Chapel Hill, University of North Carolina Press.

\section{Yates, Frances A.}

1986 [1975] L’Art de la mémoire, trad. de l'anglais par Daniel Arasse. Paris, Gallimard.

\section{Zayd, Nasr Hamid Abu}

2005 «Everyday Life, Qur'an», in Jane Dammen McAuliffe (dir.), Encyclopaedia of the Qur'an. Leiden, Brill.

\section{Zeghal, Malika}

2005 Les Islamistes marocains

le défi à la monarchie.

Paris, La Découverte.
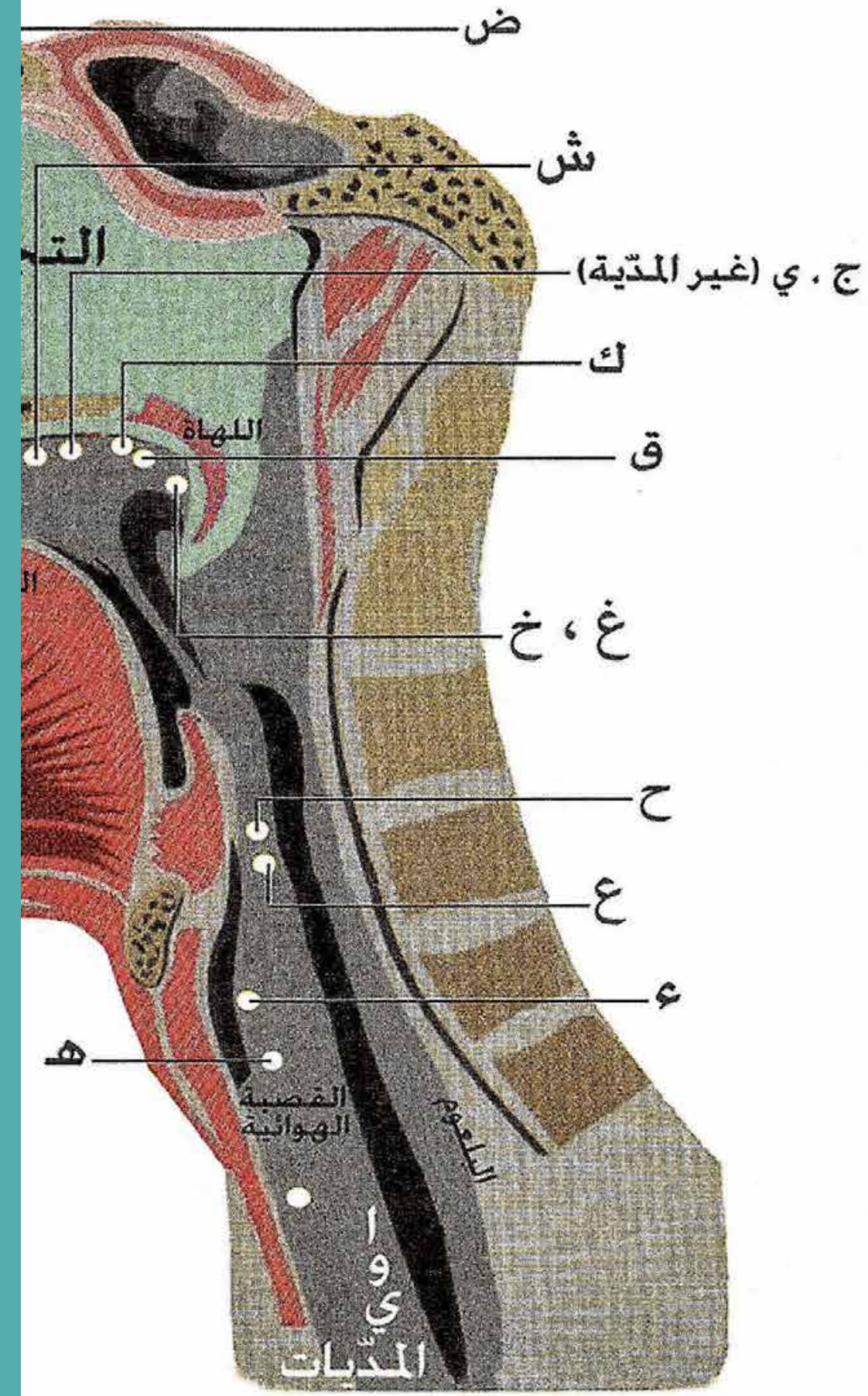